\title{
Pointwise Relations between Information and Estimation in Gaussian Noise
}

\author{
Kartik Venkat*, Tsachy Weissman*
}

\begin{abstract}
Many of the classical and recent relations between information and estimation in the presence of Gaussian noise can be viewed as identities between expectations of random quantities. These include the I-MMSE relationship of Guo et al.; the relative entropy and mismatched estimation relationship of Verdú; the relationship between causal estimation and mutual information of Duncan, and its extension to the presence of feedback by Kadota et al.; the relationship between causal and non-casual estimation of Guo et al., and its mismatched version of Weissman. We dispense with the expectations and explore the nature of the pointwise relations between the respective random quantities. The pointwise relations that we find are as succinctly stated as - and give considerable insight into - the original expectation identities.

As an illustration of our results, consider Duncan's 1970 discovery that the mutual information is equal to the causal MMSE in the AWGN channel, which can equivalently be expressed saying that the difference between the input-output information density and half the causal estimation error is a zero mean random variable (regardless of the distribution of the channel input). We characterize this random variable explicitly, rather than merely its expectation. Classical estimation and information theoretic quantities emerge with new and surprising roles. For example, the variance of this random variable turns out to be given by the causal MMSE (which, in turn, is equal to the mutual information by Duncan's result).
\end{abstract}

Index Terms

Mutual information, minimum mean square error, Brownian motion, information density, Gaussian channel, causal/filtering error, non-causal/smoothing error, Radon-Nikodym derivative, Girsanov theory, Itô calculus, scalar estimation, continuous time estimation

\section{INTRODUCTION}

The literature abounds with results that relate classical quantities in information and estimation theory. Of particular elegance are relations that have been established in the presence of additive Gaussian noise. In this work, we refine and deepen our understanding of these relations by exploring their 'pointwise' properties.

Duncan, in [1], showed that for the continuous-time additive white Gaussian noise channel, the minimum mean squared filtering(causal estimation) error is twice the input-output mutual information for any underlying signal distribution. Another discovery was made by Guo et al. in [2], where the derivative of the mutual information was

\footnotetext{
* Stanford University. Email: kvenkat@stanford.edu, tsachy@stanford.edu
} 
found to equal half the minimum mean squared error in non-causal estimation. By combining these two intriguing results, the authors of [2] also establish the remarkable equality of the causal mean squared error (at some "signal to noise' level snr) and the non-causal error averaged over 'signal to noise' ratio uniformly distributed between 0 and snr. There have been extensions of these results to the presence of mismatch. In this case, the relative entropy and the difference of the mismatched and matched mean squared errors are bridged together: Mismatched estimation in the scalar Gaussian channel was considered by Verdú in [3]. In [4], a generalization of Duncan's result to incorporate mismatch for the full generality of continuous time processes is provided. In [5], Kadota et al. generalize Duncan's theorem to the presence of feedback. These, and similar interconnections between information and estimation are quite intriguing, and merit further study of their inner workings, which is the goal of this paper .

The basic information-estimation identities, such as the ones mentioned above, can be formulated as expectation identities. We explicitly characterize the random quantities involved in a pointwise sense, and in the process elicit new connections between information and estimation for the Gaussian channel. Girsanov theory and Itô calculus provide us with tools to understand the pointwise behavior of these random quantities, and to explore their properties.

The paper is organized as follows. In Section II] we present and discuss our main results. In Section III we further develop and expand our results and observations for the setting of scalar random variables. The detailed proofs are provided in Section IV We conclude in Section $\mathrm{V}$ with a summary of our main findings.

\section{MAIN RESUlts}

\section{A. Scalar Estimation}

1) Matched Case: We begin by describing the problem setting. We are looking at mean square estimation in the presence of additive white Gaussian noise. This problem is characterized by an underlying clean signal $X$ (which follows a law $P_{X}$ ) and its AWGN corrupted version $Y_{\gamma}$ measured at a given 'signal-to-noise ratio' $\gamma$, which is to say

$$
Y_{\gamma} \mid X \sim \mathcal{N}(\sqrt{\gamma} X, 1)
$$

or equivalently

$$
Y_{\gamma} \mid X \sim \mathcal{N}(\gamma X, \gamma)
$$

where $\mathcal{N}\left(\mu, \sigma^{2}\right)$ denotes the Gaussian distribution with mean $\mu$ and variance $\sigma^{2}$.

In a communication setting, we are interested in the mutual information between the input $X$ and the output $Y_{\gamma}$. It quantifies the ability of the channel to convey useful information. In an estimation setting, one would be interested in using the observed output to estimate the underlying input signal while minimizing a given loss function. Define $\operatorname{mmse}(\gamma)$ to be the minimum mean square error at 'signal-to-noise ratio' $\gamma$

$$
\operatorname{mmse}(\gamma)=\mathrm{E}\left[\left(X-\mathrm{E}\left[X \mid Y_{\gamma}\right]\right)^{2}\right] .
$$

Intriguing ties have been discovered between the input-output mutual information and the mean squared estimation loss for the Gaussian channel. In [2], Guo et. al. discovered the I-MMSE relationship; which tells us that for the 
additive Gaussian channel, the following relationship holds between the minimum mean square error and the mutual information between input $X$ and output $Y_{\mathrm{snr}}$ (the subscript making the 'signal-to-noise ratio' explicit)

$$
\frac{d}{d \mathrm{snr}} I\left(X ; Y_{\mathrm{snr}}\right)=\frac{1}{2} \operatorname{mmse}(\mathrm{snr}) \text {. }
$$

Writing 4 in its integral form,

$$
I\left(X ; Y_{\mathrm{snr}}\right)=\frac{1}{2} \int_{0}^{\mathrm{snr}} \operatorname{mmse}(\gamma) d \gamma .
$$

Recall that we can express the mutual information between two random variables as

$$
I(X ; Y)=\mathrm{E}\left[\log \frac{d P_{Y \mid X}}{d P_{Y}}\right]
$$

where the quantity in the brackets denotes the log Radon-Nikodym derivative of the measure induced by the conditional law of $Y \mid X$ with respect to the measure induced by the law of $Y$. This quantity is referred to in some parts of the literature as the input-output information density $i(X, Y)$ (cf. [6]). In particular, let us look at the following additive Gaussian channel at 'signal-to-noise ratio' $\gamma$,

$$
Y_{\gamma}=\gamma X+W_{\gamma}
$$

for $\gamma \in[0, \mathrm{snr}]$, where $W$. is a standard Brownian motion [7], independent of $X$. Recall that $W_{\gamma} \sim \mathcal{N}(0, \gamma)$.

Now that $\left(X, Y_{0}^{\mathrm{snr}}\right)$ are on the same probability space (where throughout $Y_{0}^{s n r}$ is shorthand for $\left\{Y_{\gamma}, 0 \leq \gamma \leq\right.$ $s n r\}$ ), it is meaningful to interchange the expectation and integration in the right hand side of (5) to yield the following equivalent representation of the I-MMSE result

$$
\mathrm{E}\left[\log \frac{d P_{Y_{\mathrm{snr}} \mid X}}{d P_{Y_{\mathrm{snr}}}}\right]=\mathrm{E}\left[\frac{1}{2} \int_{0}^{\mathrm{snr}}\left(X-\mathrm{E}\left[X \mid Y_{\gamma}\right]\right)^{2} d \gamma\right]
$$

In other words, the I-MMSE relationship can be restated succinctly as:

$$
\mathrm{E}[Z]=0
$$

where

$$
Z=\log \frac{d P_{Y_{\mathrm{snr}} \mid X}}{d P_{Y_{\mathrm{snr}}}}-\frac{1}{2} \int_{0}^{\mathrm{snr}}\left(X-\mathrm{E}\left[X \mid Y_{\gamma}\right]\right)^{2} d \gamma
$$

denotes the "tracking error between the information density and half the squared error integrated over snr". But what can we say about the random variable $Z$ itself, beyond the fact that it has zero mean? Is there a crisp characterization of this random variable? The answer is captured in the following Proposition, where we present our first pointwise result.

Proposition 1: Assume $X$ has finite variance. $Z$, as defined in (10), satisfies

$$
Z=\int_{0}^{\mathrm{snr}}\left(X-\mathrm{E}\left[X \mid Y_{\gamma}\right]\right) \cdot d W_{\gamma} \quad \text { a.s. }
$$

where the integral on the right hand side of 11 denotes the Itô integral with respect to $W$.. 
In particular, the above characterization implies that $Z$ is a martingale, and (by virtue of having zero expectation) directly implies the I-MMSE relationship in (9) (which is equivalent to (5)). Another immediate consequence of Proposition 1 is the following:

Theorem 1: Assume $X$ has finite variance. Then

$$
\operatorname{Var}(Z)=\int_{0}^{\mathrm{snr}} \operatorname{mmse}(\gamma) d \gamma=2 I\left(X ; Y_{\mathrm{snr}}\right)
$$

Thus we observe a simple characterization of the second moment of the tracking error, in terms of classical estimation and information quantities. The relationship in (12) tells us how far apart the information density and the estimation error typically are, two quantities that we know to have equal expectations - and in particular that the variance of their difference can be described directly in terms of the original estimation error.

2) Mismatched Case: We now turn to the scenario of mismatched estimation, where the underlying clean signal $X$ is distributed according to $P$, while the decoder believes the law to be $Q$. [3] presents the following relationship between the relative entropy of the true and mismatched output laws, and the difference between the mismatched and matched estimation losses:

$$
D(P * \mathcal{N}(0,1 / \mathrm{snr}) \| Q * \mathcal{N}(0,1 / \mathrm{snr}))=\frac{1}{2} \int_{0}^{\mathrm{snr}} m s e_{P, Q}(\gamma)-m s e_{P, P}(\gamma) d \gamma,
$$

where $*$ denotes the convolution operation, and $m s e_{P, Q}(\gamma)$ is defined as

$$
\operatorname{mse}_{P, Q}(\gamma)=\mathrm{E}_{P}\left[\left(X-\mathrm{E}_{Q}\left[X \mid Y_{\gamma}\right]\right)^{2}\right] .
$$

Towards deriving a pointwise extension of (13), we note that it can be recast, assuming again the observation model in (7), as the expectation identity

$$
\mathrm{E}\left[\log \frac{d P_{Y_{\mathrm{snr}}}}{d Q_{Y_{\mathrm{snr}}}}\right]=\mathrm{E}\left[\frac{1}{2} \int_{0}^{\mathrm{snr}}\left(X-\mathrm{E}_{Q}\left[X \mid Y_{\gamma}\right]\right)^{2}-\left(X-\mathrm{E}_{P}\left[X \mid Y_{\gamma}\right]\right)^{2} d \gamma\right] .
$$

Let $Z_{M}$ denote the difference between the random quantities appearing in the above expression, i.e.

$$
Z_{M}=\log \frac{d P_{Y_{\mathrm{snr}}}}{d Q_{Y_{\mathrm{snr}}}}-\frac{1}{2} \int_{0}^{\mathrm{snr}}\left(X-\mathrm{E}_{Q}\left[X \mid Y_{\gamma}\right]\right)^{2}-\left(X-\mathrm{E}_{P}\left[X \mid Y_{\gamma}\right]\right)^{2} d \gamma
$$

In the following, we provide an explicit characterization of this random variable.

Proposition 2: Assuming $X$ has finite variance under both $P$ and $Q, Z_{M}$ defined in [16, satisfies

$$
Z_{M}=\int_{0}^{\mathrm{snr}}\left(\mathrm{E}_{P}\left[X \mid Y_{\gamma}\right]-\mathrm{E}_{Q}\left[X \mid Y_{\gamma}\right]\right) \cdot d W_{\gamma} \quad P-a . s .
$$

We observe that the above Itô integral is a martingale and consequently has zero expectation $\mathrm{E}\left[Z_{M}\right]=0$, recovering [15], i.e.Verdú's relation from [3]. A further implication that can be read off of Proposition 2 rather immediately (as will be explicitly shown in the Section IV] is the following:

Theorem 2: Assuming $X$ has finite variance under both $P$ and $Q, Z_{M}$ defined in [16, satisfies

$$
\operatorname{Var}\left(Z_{M}\right)=\int_{0}^{\mathrm{snr}} m s e_{P, Q}(\gamma)-m s e_{P, P}(\gamma) d \gamma=2 D(P * \mathcal{N}(0,1 / \mathrm{snr}) \| Q * \mathcal{N}(0,1 / \mathrm{snr}))
$$


Similarly as in the non-mismatched case, we observe that the variance of the difference between the information and estimation theoretic random variables whose expectations comprise the respective two sides of Verdú's mismatch relationship has a distribution independent characterization in terms of the matched and mismatched estimation errors and consequently, by yet another application of this same relationship of Verdú, in terms of the relative entropy between the output distributions. In the following subsection we extend this line of inquiry and results from the scalar case to that where the channel input is a continuous-time process.

\section{B. Continuous Time}

We now turn to the continuous-time Gaussian channel. Let $X_{0}^{T}$ be the underlying noise-free process (with finite power) to be estimated. The continuous time channel is characterized by the following relationship between the input and output processes,

$$
d Y_{t}=X_{t} d t+d W_{t}
$$

where $\left\{W_{t}\right\}_{t \geq 0}$ is a standard Brownian motion, independent of $X_{0}^{T}$.

1) "Pointwise Duncan": In [1], Duncan proved the equivalence of input-output mutual information to the filtering squared error, of a finite powered continuous time signal $X_{t}$, corrupted according to 19 to yield the noise corrupted process $Y_{t}$. The signal is observed for a time duration $[0, T]$. Denoting the time averaged filtering squared error,

$$
\operatorname{cmmse}(T)=\int_{0}^{T} E\left[\left(X_{t}-E\left[X_{t} \mid Y^{t}\right]\right)^{2}\right] d t
$$

and letting $I\left(X^{T} ; Y^{T}\right)$ denote the input-output mutual information, Duncan's theorem then tells us that,

$$
I\left(X^{T} ; Y^{T}\right)=\frac{1}{2} \mathrm{cmmse}(T) .
$$

In [5], Kadota et al. extend this result to communication over channels with feedback, and in the recent [8] this result is extended to more general scenarios involving the presence of feedback, and it is shown that 21] remains true in these more general cases upon replacing the mutual information on the left hand side with directed information. In [9], several properties of likelihood ratios and their relationships with estimation error are studied. We now proceed to describe a pointwise characterization of Duncan's theorem.

Considering the random variable $D(T)$ defined as

$$
D(T)=\log \frac{d P_{Y^{T} \mid X^{T}}}{d P_{Y^{T}}}-\frac{1}{2} \int_{0}^{T}\left(X_{t}-E\left[X_{t} \mid Y^{t}\right]\right)^{2} d t
$$

Duncan's theorem is equivalently expressed as

$$
E[D(T)]=0
$$

We now present an explicit formula for $D(T)$ in the following Proposition.

Proposition 3: Let $D(T)$ be as defined in 22,. Then,

$$
D(T)=\int_{0}^{T}\left(X_{t}-E\left[X_{t} \mid Y^{t}\right]\right) d W_{t} \quad \text { a.s.. }
$$


Note that on the the right side of 24] is a stochastic integral with respect to the Brownian motion $W$. driving the noise in the channel. With this representation, Duncan's theorem follows from the mere fact that this stochastic integral is a martingale and, in particular, has zero expectation.

On applying another basic property of the stochastic integral we get the following interesting result for the variance of $D(T)$.

Theorem 3: For a continuous-time signal $X_{0}^{T}$ with finite power, $D(T)$ as defined in 22] satisfies

$$
\operatorname{Var}(D(T))=\mathrm{cmmse}(T) .
$$

In conjunction with Duncan's theorem [21, we get the following relationship,

$$
\operatorname{Var}(D(T))=\mathrm{cmmse}(T)=2 I\left(X^{T} ; Y^{T}\right)
$$

which parallels our discovery for scalar random variables, in (12). Thus, we find that the pointwise tracking error satisfies this intriguing distribution independent property, for the full generality of continuous time inputs for the Gaussian Channel. That the estimation error and mutual information emerge from this analysis in such a crisp manner is quite satisfying.

Remark 1: One can note that for $X_{t} \equiv X$ in the interval $[0, T]$, we can use the results in 25] and 24], to recover Theorem 1 and its its pointwise characterization in Proposition 1 respectively.

Among the additional immediate benefits the characterization in (24), is that it allows us to infer facts about the limiting behavior of the random variables involved, such as in the following theorem.

Theorem 4: Suppose that the process $\left\{X_{t}\right\}_{t \geq 0}$ satisfies

$$
\lim _{T \rightarrow \infty} \frac{1}{T^{2}} \mathrm{cmmse}(T)=0
$$

(or, equivalently, by Duncan's theorem, $\lim _{T \rightarrow \infty} I\left(X^{T} ; Y^{T}\right) / T^{2}=0$ ). Then,

$$
\text { l.i.m. } T \rightarrow \infty \frac{1}{T}\left[\log \frac{d P_{Y_{0}^{T} \mid X_{0}^{T}}}{d P_{Y_{0}^{T}}}-\frac{1}{2} \int_{0}^{T}\left(X_{t}-\mathrm{E}\left[X_{t} \mid Y_{0}^{t}\right]\right)^{2} d t\right]=0,
$$

where 1.i.m. denotes Limit in the Mean.

We already know from Duncan's theorem that the two quantities that make up $D(T)$, namely the information density and the causal estimation error, are equal in expectation for every $T>0$, but our formulation reveals much more about the pointwise behavior of these random quantities in themselves. In particular, it is interesting to note that so little is needed to guarantee the convergence in 28): not even wide sense stationarity of the marginal of the underlying process is required. Indeed, any process with $\operatorname{Var}\left(X_{t}\right)$ growing sublinearly with $t$ is easily seen to satisfy 27].

2) Pointwise Mismatch: We now consider the setting in [4], where a continuous time signal $X_{t}$, distributed according to a law $P$ is observed through additive Gaussian noise, and is estimated by an estimator that would have been optimal if the signal had followed the law $Q$. In this general setting, the main result in [4] shows that the relative entropy between the laws of the output for the two different underlying distributions ( $\mathrm{P}$ and $\mathrm{Q})$, is exactly half the difference between the mismatched and matched filtering errors. Let $Y_{t}$ be the continuous time AWGN 
corrupted version of $X_{t}$ as given by 19$]$. Let $P_{Y_{0}^{T}}$ and $Q_{Y_{0}^{T}}$ be the output distributions when the underlying signal $X_{0}^{T}$ has law $P$ and $Q$, respectively. As before, T denotes the time duration for which the process is observed. We denote the mismatched causal mean squared error,

$$
\text { cmse }_{P, Q}(T)=\int_{0}^{T} E_{P}\left[\left(X_{t}-E_{Q}\left[X_{t} \mid Y^{t}\right]\right)^{2}\right] d t .
$$

In this setting, [4] tells us that the relative entropy between the output distributions is half the difference between the mismatched and matched filtering errors, i.e.

$$
D\left(P_{Y_{0}^{T}} \| Q_{Y_{0}^{T}}\right)=\frac{1}{2}\left[\operatorname{cmse}_{P, Q}(T)-\operatorname{cmse}_{P, P}(T)\right]
$$

Define the pointwise difference between the log Radon-Nikodym derivative and half the mismatched causal squared error difference,

$$
M(T)=\log \frac{d P_{Y_{0}^{T}}}{d Q_{Y_{0}^{T}}}-\frac{1}{2} \int_{0}^{T}\left[\left(\mathrm{E}_{Q}\left[X_{t} \mid Y^{t}\right]-X_{t}\right)^{2}-\left(\mathrm{E}_{P}\left[X_{t} \mid Y^{t}\right]-X_{t}\right)^{2}\right] d t
$$

Note that according to the above definition, 30 can be equivalently stated as

$$
\mathrm{E}[M(T)]=0 .
$$

But in fact much more can be said about $M(T)$ :

Proposition 4:

$$
M(T)=\int_{0}^{T}\left(\mathrm{E}_{P}\left[X_{t} \mid Y^{t}\right]-\mathrm{E}_{Q}\left[X_{t} \mid Y^{t}\right]\right) d W_{t} \quad P-\text { a.s. }
$$

where $M(T)$ is as defined in (31), and $X_{t}$ is assumed to have finite power under the laws $P$ as well as $Q$.

We note that relation (30) is implied immediately by Proposition 4 due to the 'zero mean' property of the martingale $M(T)$. But more can be read off of this result. For example, the following characterization of the variance of $M(T)$ will be shown in Section IV to follow quite directly from Proposition 4

Theorem 5: $M(T)$ as defined in 30, satisfies

$$
\operatorname{Var}(M(T))=\operatorname{cmse}_{P, Q}(T)-\operatorname{cmse}_{P, P}(T)=2 D\left(P_{Y_{0}^{T}} \| Q_{Y_{0}^{T}}\right)
$$

Thus, the variance of $M(T)$ is exactly the difference between the causal mismatched and matched squared errors. And further, from [4] we know that it is equal to twice the relative entropy between the output distributions according to laws $P$ and $Q$.

3) Presence of Feedback: In the previous subsections, we explicitly characterized a pointwise relationship between the log Radon-Nikodym derivates associated with the informational quantities and the squared filtering error. These characterizations give us a crisp understanding of well known information-estimation results such as Duncan's theorem [1], and the equivalence between mismatched estimation and relative entropy [4] for the continuous time setting. These results emerge as direct corollaries of our characterization of the tracking errors as stochastic integrals. Further, we establish a new equivalence between estimation error and variance of the tracking error. 
In this section we revisit [5], where Kadota et al. present a generalization of Duncan's theorem for the additive Gaussian channel with feedback. The channel input $\phi_{t}$ is a function of the underlying process $X_{t}$ as well as the past outputs of the channel $Y_{0}^{t}$, in an additive Gaussian noise setting. The observation window is $t \in[0, T]$. The channel can be represented as,

$$
Y_{t}=\int_{0}^{t} \phi_{s}\left(Y_{0}^{s}, X_{s}\right) d s+W_{t},
$$

where, as usual, the standard Brownian motion $W$. is independent of the underlying process $X_{(\cdot)}$. In differential form, (and using shorthand to represent $\phi_{t}\left(Y_{0}^{t}, X_{t}\right)$ ) we can rewrite 35 as

$$
d Y_{t}=\phi_{t} d t+d W_{t}
$$

We denote the causal estimate of $\phi_{t}$ based on observations up until $t$ by

$$
\hat{\phi}_{t}=E\left[\phi_{t} \mid Y_{0}^{t}\right]
$$

Under mild regularity conditions on $\phi_{t}$, the mutual information between the input and output is equal to half the causal mean squared error. With our notation, the main result of [5] is expressed as

$$
I\left(X_{0}^{T} ; Y_{0}^{T}\right)=\frac{1}{2} \int_{0}^{T} E\left[\left(\phi_{t}-\hat{\phi}_{t}\right)^{2}\right] d t
$$

We use Girsanov theory to develop a pointwise relationship in this setting, akin to our treatment of Duncan's theorem. Defining

$$
D_{\phi}(T) \triangleq \log \frac{d P_{Y^{T} \mid X^{T}}}{d P_{Y^{T}}}-\frac{1}{2} \int_{0}^{T}\left(\phi_{t}-\hat{\phi}_{t}\right)^{2} d t
$$

We have the following:

Theorem 6: For $\phi_{t}$ which satisfy a finite power criterion, we have

$$
D_{\phi}(T)=\int_{0}^{T}\left(\phi_{t}-\hat{\phi}_{t}\right) d W_{t} \quad \text { a.s. }
$$

where $D_{\phi}(T)$ is as defined in 39 .

Parallel to our discovery in the pointwise treatment of Duncan's theorem, we can use Theorem 6 to deduce various results. Note from 40, that $D_{\phi}(T)$ is a martingale. Therefore,

$$
\mathrm{E}\left[D_{\phi}(T)\right]=0
$$

recovering the main result of [5], namely [38]. Using Itô's Isometry we also immediately obtain:

Corollary 7:

$$
\begin{aligned}
\operatorname{Var}\left(D_{\phi}(T)\right) & =\int_{0}^{T} E\left[\left(\phi_{t}-\hat{\phi}_{t}\right)^{2}\right] d t \\
& =\operatorname{cmmse}_{\phi}(T)
\end{aligned}
$$

The above follows directly from the application of Itô's Isometry property to the stochastic integral in 40 and noting that $\mathrm{E}\left[D_{\phi}(T)\right]=0$. 
Thus, even for the generalized setting of communication over channels with feedback, we can characterize how closely the information density and squared filtering error track each other. We note that the second moment of the tracking error is equal to the filtering error for all finite powered distributions of the underlying signal. In particular, these results may have applications in approximating the mutual information via estimation theoretic quantities, for channels with feedback. In the special case when $\phi_{t}=X_{t}$, we recover the results obtained in the pointwise treatment of Duncan's theorem in Section [II-B1] Here, we would like to note that Theorem 6 can further be extended to accommodate mismatch.

Let us denote separately, the Causal Estimates of $\phi_{t}$ under the two laws $P$ and $Q$ that govern the underlying process $X_{t}$ :

$$
\begin{aligned}
& \hat{\phi}_{t}^{P}=\mathrm{E}_{P}\left[\phi_{t} \mid Y_{0}^{t}\right] \\
& \hat{\phi}_{t}^{Q}=\mathrm{E}_{Q}\left[\phi_{t} \mid Y_{0}^{t}\right]
\end{aligned}
$$

Define,

$$
M_{\phi}(T) \triangleq \log \frac{d P_{Y_{0}^{T}}}{d Q_{Y_{0}^{T}}}-\frac{1}{2} \int_{0}^{T}\left[\left(\hat{\phi}_{t}^{Q}-\phi_{t}\right)^{2}-\left(\hat{\phi}_{t}^{P}-\phi_{t}\right)^{2}\right] d t .
$$

As will be shown in the next section, Girsanov theory allows us to establish, for this setting

$$
D\left(P_{Y_{0}^{T}} \| Q_{Y_{0}^{T}}\right)=\frac{1}{2}\left[c m s e_{P, Q}^{\phi}(T)-\operatorname{cmse}_{P, P}^{\phi}(T)\right],
$$

where

$$
\operatorname{cmse}_{P, Q}^{\phi}(T)=\int_{0}^{T} \mathrm{E}_{P}\left[\left(\phi_{t}-\hat{\phi}_{t}^{Q}\right)^{2}\right] d t
$$

Define,

$$
M_{\phi}(T)=\log \frac{d P_{Y_{0}^{T}}}{d Q_{Y_{0}^{T}}}-\frac{1}{2} \int_{0}^{T}\left[\left(\hat{\phi}_{t}^{Q}-\phi_{t}\right)^{2}-\left(\hat{\phi}_{t}^{P}-\phi_{t}\right)^{2}\right] d t .
$$

The arguments given in the proof of Proposition 4 and the treatment of the non-mismatched case above, can be carried over to show the following:

Theorem 8: For a finite power constraint on $\phi_{t}$ under laws $P$ and $Q, M_{\phi}(T)$ as defined in 49p satisfies

$$
M_{\phi}(T)=\int_{0}^{T}\left(\hat{\phi}_{t}^{P}-\hat{\phi}_{t}^{Q}\right) d W_{t} \quad P-\text { a.s. }
$$

Note that 47 follows directly from Theorem 8 , by noting that $M_{\phi}(T)$ is a martingale and consequently has zero mean. Thus, the generalized D-MSE relationship for channels with feedback is an expectation identity that arises from the pointwise treatment of the tracking error in 49p. As a corollary, we also obtain the generalized result for the second moment of the tracking error, which acts as a bridge between the relative entropy and the difference of the mismatched and matched filtering errors,

Corollary 9:

$$
\operatorname{Var}\left(M_{\phi}(T)\right)=c m s e_{P, Q}^{\phi}(T)-c m s e_{P, P}^{\phi}(T)=2 D\left(P_{Y_{0}^{T}} \| Q_{Y_{0}^{T}}\right)
$$


The result in 51) can be specialized to obtain results similar to those obtained previously for "Pointwise Duncan", and pointwise mismatch in the absence of feedback.

4) Pointwise I-MMSE for processes: In [2], Guo et al. present what is known as the I-MMSE relationship for the Gaussian channel. This result states that the mutual information (at signal-to-noise ratio level 'snr') is the integral over SNR (from level 0 to 'snr') of half the non-causal squared error.

In this subsection, we present a characterization of the pointwise nature of the I-MMSE relationship for processes in the continuous time Gaussian channel. We first explain the channel model. Since we are now concerned with two continuously varying parameters, namely time and 'snr', the Gaussian noise corrupting the signal is a standard Brownian sheet $W_{t, \gamma}$. For a fixed $\gamma$, we let $W^{(\gamma)}$ denote the Brownian motion defined by $W_{t}^{(\gamma)}=W_{t, \gamma}$. The channel then, at $\operatorname{SNR} \gamma$, is given by

$$
d Y_{t}^{(\gamma)}=\gamma X_{t} d t+d W_{t}^{(\gamma)}
$$

where $\left\{X_{t}\right\}_{0 \leq t \leq T}$ is the underlying noise free process, which is independent of the Brownian sheet. The output of the channel at SNR $\gamma$, is denoted by $Y_{0}^{T,(\gamma)}=\left\{Y_{t}^{(\gamma)}\right\}_{0 \leq t \leq T}$. In this framework, the I-MMSE relationship from [2] tells us that,

$$
I\left(X_{0}^{T} ; Y_{0}^{T,(\mathrm{snr})}\right)=\frac{1}{2} \int_{0}^{\mathrm{snr}} \operatorname{mmse}(\gamma) d \gamma
$$

where

$$
\operatorname{mmse}(\gamma)=\int_{0}^{T} \mathrm{E}\left[\left(X_{t}-\mathrm{E}\left[X_{t} \mid Y_{0}^{T,(\gamma)}\right]\right)^{2}\right] d t .
$$

Note that 53 can now equivalently be stated as ,

$$
\mathrm{E}\left[\log \frac{d P_{Y_{0}^{T,(s n r)} \mid X_{0}^{T}}}{d P_{Y_{0}^{T,(\text { snr })}}}\right]=\mathrm{E}\left[\frac{1}{2} \int_{0}^{\mathrm{snr}} \int_{0}^{T}\left(X_{t}-\mathrm{E}\left[X_{t} \mid Y_{0}^{T,(\gamma)}\right]\right)^{2} d t d \gamma\right]
$$

Defining the pointwise difference between the input-output information density and half the non-causal error integrated over time and SNR

$$
N(T) \triangleq \log \frac{d P_{Y_{0}^{T,(\gamma)} \mid X_{0}^{T}}}{d P_{Y_{0}^{T,(\gamma)}}}-\frac{1}{2} \int_{0}^{\mathrm{snr}} \int_{0}^{T}\left(X_{t}-\mathrm{E}\left[X_{t} \mid Y_{0}^{T,(\gamma)}\right]\right)^{2} d t d \gamma,
$$

we first note that the I-MMSE relationship in (55) can equivalently be stated as

$$
\mathrm{E}[N(T)]=0 .
$$

In doing so, we formulate the I-MMSE relationship as an expectation identity. In the previous discussions, we observed an estimation theoretic flavor to the second moment of the tracking error in characterizations of both Duncan's result and that of mismatched estimation. This kind of a relationship turns out to hold also in our present context:

Theorem 10: For a finite power continuous-time process $X^{T}, N(T)$ as defined in 56 satisfies

$$
\operatorname{Var}(N(T))=\int_{0}^{\mathrm{snr}} \operatorname{mmse}(\gamma) d \gamma=2 I\left(X_{0}^{T} ; Y_{0}^{T,(\mathrm{snr})}\right) .
$$


In establishing the above result in the Section IV, we use a multidimensional version of Girsanov's theorem to characterize the input-output information density of a piecewise constant process observed through AWGN. We then use approximation arguments to extend the result to the class of finite power continuous time processes.

5) Pointwise causal vs. non-causal error: By a combination of Duncan's theorem and the I-MMSE result, the authors of [2] establish the equivalence of the causal error at SNR level 'snr', and the non-causal error averaged over SNR uniformly distributed between 0 and 'snr'. The input-output mutual information acts as a bridge between the quantities. Let 'cmmse(snr)' denote the integral of the filtering error for the channel described in 52,

$$
\text { cmmse }(\mathrm{snr})=\int_{0}^{T} \mathrm{E}\left[\left(X_{t}-\mathrm{E}\left[X_{t} \mid\left\{Y_{s}^{(\mathrm{snr})}\right\}_{0 \leq s \leq t}\right]\right)^{2}\right] d t .
$$

Recalling the definition of the non-causal error ' $m m s e(\gamma)$ ' in (54), the causal vs. non-causal error relationship is

$$
\mathrm{cmmse}(\mathrm{snr})=\frac{1}{\mathrm{snr}} \int_{0}^{\mathrm{snr}} \operatorname{mmse}(\gamma) d \gamma
$$

So far, we have presented pointwise characterizations of Duncan's result in Section II-B1 as well as the I-MMSE relationship for continuous time processes in Section [II-B4. Using these two characterizations, in Section IV] we develop a pointwise version of the celebrated estimation-theoretic result 60. Specifically, we show that under the Brownian sheet induced channel described in 52, and under some regularity assumed on the process $X^{T}$, the difference

$$
\int_{0}^{T}\left(X_{t}-E\left[X_{t} \mid Y_{0}^{t,(s n r)}\right]\right)^{2} d t-\frac{1}{s n r} \int_{0}^{s n r}\left[\int_{0}^{T}\left(X_{t}-E\left[X_{t} \mid Y_{0}^{T,(\gamma)}\right]\right)^{2} d t\right] d \gamma
$$

can be characterized as a difference between stochastic integrals. In particular, such a characterization immediately implies 60).

6) Pointwise causal vs. anticausal error: Now, we present another interesting application of Proposition 3 . a pointwise treatment of the causal vs. anti-causal estimation error relationship. Duncan's theorem gives us the remarkable equality between the causal squared error and input-output mutual information for the continuous-time Gaussian channel. Invoking the invariance of mutual information to the direction of time, it can be observed (as is noted in [2]) that the causal squared error is equal to the anticausal squared error (for a given 'snr'), regardless of the input distribution of the underlying process. Let $X_{t}$ be the noise free stochastic process that is distributed according to law P. Let $Y_{t}$ be the continuous time AWGN corrupted version of $X_{t}$ at $s n r=1$, according to [19]. Let the observation window be $t \in[0, T]$.

Let us now denote

$$
\begin{aligned}
\tilde{X}_{t} & =X_{T-t}, \\
\tilde{Y}_{t} & =Y_{T}-Y_{T-t}, \\
B_{t} & =W_{T}-W_{T-t} .
\end{aligned}
$$

Note that the anti-causal estimation error for the original processes $\left(X^{T}, Y^{T}\right)$ is given by the causal estimation error associated with these "tilded" processes $\left(\tilde{X}^{T}, \tilde{Y}^{T}\right)$, i.e. :

$$
\int_{0}^{T} E\left[\left(\tilde{X}_{t}-\mathrm{E}\left[\tilde{X}_{t} \mid \tilde{Y}_{0}^{t}\right]\right)^{2}\right] d t=\int_{0}^{T} E\left[\left(X_{t}-\mathrm{E}\left[X_{t} \mid Y_{t}^{T}\right]\right)^{2}\right] d t
$$


Define the difference of the causal and anti-causal estimation errors,

$$
J(T) \triangleq \int_{0}^{T}\left(X_{t}-\mathrm{E}\left[X_{t} \mid Y_{0}^{t}\right]\right)^{2} d t-\int_{0}^{T}\left(\tilde{X}_{t}-\mathrm{E}\left[\tilde{X}_{t} \mid \tilde{Y}_{0}^{t}\right]\right)^{2} d t
$$

Then, we have the following pointwise result relating the difference of the causal and anti-causal errors

Proposition 5: For a process $X^{T}$ with finite power, $J(T)$ as defined in 66 satisfies

$$
\frac{1}{2} J(T)=\int_{0}^{T} E\left[X_{t} \mid Y_{0}^{t}\right] \cdot d W_{t}-\int_{0}^{T} E\left[\tilde{X}_{t} \mid \tilde{Y}_{0}^{t}\right] \cdot d B_{t} \quad \text { a.s. }
$$

We note that the right hand side of equation 67) is the difference of two martingales. Taking expectation on both sides, we recover the equality of causal and anti-causal squared error,

$$
\int_{0}^{T} \mathrm{E}\left[\left(X_{t}-\mathrm{E}\left[X_{t} \mid Y_{0}^{t}\right]\right)^{2} d t\right]=\int_{0}^{T} \mathrm{E}\left[\left(\tilde{X}_{t}-\mathrm{E}\left[\tilde{X}_{t} \mid \tilde{Y}_{0}^{t}\right]\right)^{2}\right] d t
$$

using merely the fact that the Itô integrals on the right hand side of 67) have zero mean. Thus, we provide a pointwise characterization of the causal vs. anti-causal errors. Duncan's theorem implies, what is otherwise a surprising result, that the causal and anti-causal squared errors are equal, as rederived in (68). Through 67), we uncover the structure of and dependence between the random quantities involved and characterize their difference as a difference of two zero mean stochastic integrals.

\section{Scalar Setting: Examples, Alternative Couplings, Further Observations, AND IDENTITIES}

Returning to the scalar channel setting of Subsection II-A, we introduce notation as follows:

Definition 1:

$$
\begin{aligned}
I_{1} & =\log \frac{d P_{Y_{\mathrm{snr}} \mid X}}{d P_{Y_{\mathrm{snr}}}} \\
I_{2} & =\frac{1}{2} \int_{0}^{\mathrm{snr}}\left(X-\mathrm{E}\left[X \mid Y_{\gamma}\right]\right)^{2} d \gamma \\
Z & =I_{1}-I_{2},
\end{aligned}
$$

where $Z$, as in the previous section, is informally referred to as the "tracking error"

\section{A. The Original Coupling}

We studied the example of the scalar Gaussian channel corrupted by additive Gaussian noise where the additive noise components for the different SNR levels were coupled via a standard Brownian motion, as in (7). We characterized explicitly in Proposition 1 , the tracking error $Z$ between the information density and half the estimation error.

To illustrate how explicit this characterization allows us to be, let us consider the case of $X \sim \mathcal{N}(0,1)$, and use Proposition 11 to characterize the distribution of the random variable $Z$. Note that in this case,

$$
\mathrm{E}\left[X \mid Y_{\gamma}\right]=\frac{\gamma X+W_{\gamma}}{\gamma+1}
$$


Combining with (11), we have

$$
Z=\int_{0}^{\mathrm{snr}} \frac{X+W_{\gamma}}{\gamma+1} d W_{\gamma} \quad \text { a.s. }
$$

Also, invoking the result in Theorem [1, we obtain the variance of $Z$ to be

$$
\begin{aligned}
\operatorname{Var}(Z) & =2 I\left(X ; Y_{\text {snr }}\right) \\
& =\log (1+\text { snr })
\end{aligned}
$$

We shall now look at the pointwise scalar estimation problem in a new light. Recall that in moving from 5 to (8), we place all the random variables $\left(X, Y_{0}^{\mathrm{snr}}\right)$ on the same probability space, via a standard Brownian motion, as in (7). Note, however, that the only assumption for the original results that hold in expectation is that, for each $\gamma>0$, the channel satisfies $[1$, i.e.

$$
Y_{\gamma} \mid X \sim \mathcal{N}(\sqrt{\gamma} X, 1)
$$

where $\mathcal{N}\left(\mu, \sigma^{2}\right)$ denotes the Gaussian distribution with mean $\mu$ and variance $\sigma^{2}$. Taking the channel noise variables for the various SNR levels to be the components of a Brownian motion, as in (7), is but one possibility for a coupling that respects $(76)$.

For $Z$ as defined in $(71)$, the I-MMSE relationship tells us that $\mathrm{E}[Z]=0$, for all such $\left(X, Y_{0}^{\text {snr }}\right)$ that are consistent with (76). It is instructive to note that $(76)$ is a requirement on the channel for the individual SNR levels. As mentioned, however, there are several ways in which we can couple the input $X$ and outputs $\left\{Y_{0}^{\text {snr }}\right\}$ together so that they satisfy 76 . The I-MMSE relationship implies that for all such couplings we have $\mathrm{E}[Z]=0$. Before exploring some other examples of such 'couplings' and their properties, let us note a refinement of this zero-mean property pertaining to the random variable $Z$, which holds regardless of the coupling.

Proposition 6: Suppose $X$ has finite variance and that $Z$ is defined as in Definition 1 , under a joint distribution on $\left(X, Y_{0}^{s n r}\right)$ satisfying $(76)$. Then,

$$
\mathrm{E}[Z \mid X]=0, \quad \text { a.s. }
$$

Thus, not only is the tracking error a zero-mean random variable, but even its conditional expectation $\mathrm{E}[Z \mid X]$ is zero. We use the setting and results in [3] to establish this result. The I-MMSE relationship which states that $\mathrm{E}[Z]$ $=0$, is then immediately implied by Proposition 6 .

Having briefly touched upon the idea of ways other than the channel in (7), in which we can comply with the marginal channel requirements in (76, let us look at some concrete examples and draw a comparison between them.

\section{B. Additive Standard Gaussian}

An alternative coupling between $X$ and $Y_{\gamma}$ that respects (76), is achieved by using a scaled standard Gaussian random variable as additive noise, instead of the Brownian motion considered in the previous setting. The channel 
is described by letting, for $\gamma \in[0, \mathrm{snr}]$,

$$
Y_{\gamma}=\sqrt{\gamma} X+N
$$

where $N \sim \mathcal{N}(0,1)$ is independent of $X$. Note that in this coupling, the channel has the same noise component for all values of $\gamma$. We now present the pointwise characterization of the 'tracking error' for this setting in the following Lemma.

Lemma 11: Let $X \sim P_{X}$, have a finite second moment. For the channel in (78), the pointwise tracking error $Z$ defined in Definition 1 can be expressed as,

$$
Z=\int_{0}^{\mathrm{snr}} \tilde{Z}_{\gamma} d \gamma
$$

where $\tilde{Z}_{\gamma}$ is given by

$$
\tilde{Z}_{\gamma}=\frac{1}{2}\left\{\mathrm{E}\left[X^{2} \mid Y_{\gamma}\right]-X \mathrm{E}\left[X \mid Y_{\gamma}\right]-\left(X-\mathrm{E}\left[X \mid Y_{\gamma}\right]\right)^{2}+\frac{Y_{\gamma}}{\sqrt{\gamma}}\left(X-\mathrm{E}\left[X \mid Y_{\gamma}\right]\right)\right\} .
$$

As a sanity check, one can observe that $\mathrm{E}\left[\tilde{Z}_{\gamma} \mid Y_{\gamma}\right]=0$ and thus $\mathrm{E}\left[\tilde{Z}_{\gamma}\right]=0$. Consequently, $\mathrm{E}[Z]=\int_{0}^{\text {snr }} \mathrm{E}\left[\tilde{Z}_{\gamma}\right] d \gamma=0$. Lemma 11 is closely related to the pointwise identity in [10, Theorem 2.3]. However, for completeness we present a stand-alone proof in Section IV

Example 1: Applying Lemma11 in the case where the channel input $X$ is standard normal, i.e., $X \sim \mathcal{N}(0,1)$, the tracking error is given by

$$
Z=\frac{1}{2}\left[\log (1+\mathrm{snr})-N^{2} \log (1+\mathrm{snr})+2 X N \tan ^{-1}(\sqrt{\mathrm{snr}})\right] \quad \text { a.s. }
$$

and thus, in particular, the variance of the tracking error is

$$
\operatorname{Var}(Z)=\frac{1}{2}(\log (1+\mathrm{snr}))^{2}+\left(\tan ^{-1}(\sqrt{\mathrm{snr}})\right)^{2}
$$

For snr=1, we present a plot of the Cumulative Distribution Function of the random variable $Z$ in 81 in Figure 1 .

\section{Independent Standard Gaussian's}

We present yet another illustration of a different coupling which places the input and outputs of the Gaussian channel 76 on the same probability space. Unlike the previous two examples in Sections III-A and III-B respectively, we here look at the limiting behavior of a family of couplings achieved by the construction described below.

Let $\Delta>0$. Define $\Delta=\frac{\mathrm{snr}}{M}$ for $M$ natural. Let $\mathcal{I}_{i} \equiv[(i-1) \Delta, i \Delta)$ for $i \in\{1,2,3 \ldots M\}$. Let $N_{i}$ be independent standard Gaussian random variables $\sim \mathcal{N}(0,1)$. Now we define the following process

$$
Y_{\gamma}=\sqrt{\gamma} X+N_{\gamma}
$$

where $N_{\gamma}=N_{i}$ for $\gamma \in \mathcal{I}_{i}$. Note that this is a coupling of the channel noise components at different SNR's that adheres to (76). 


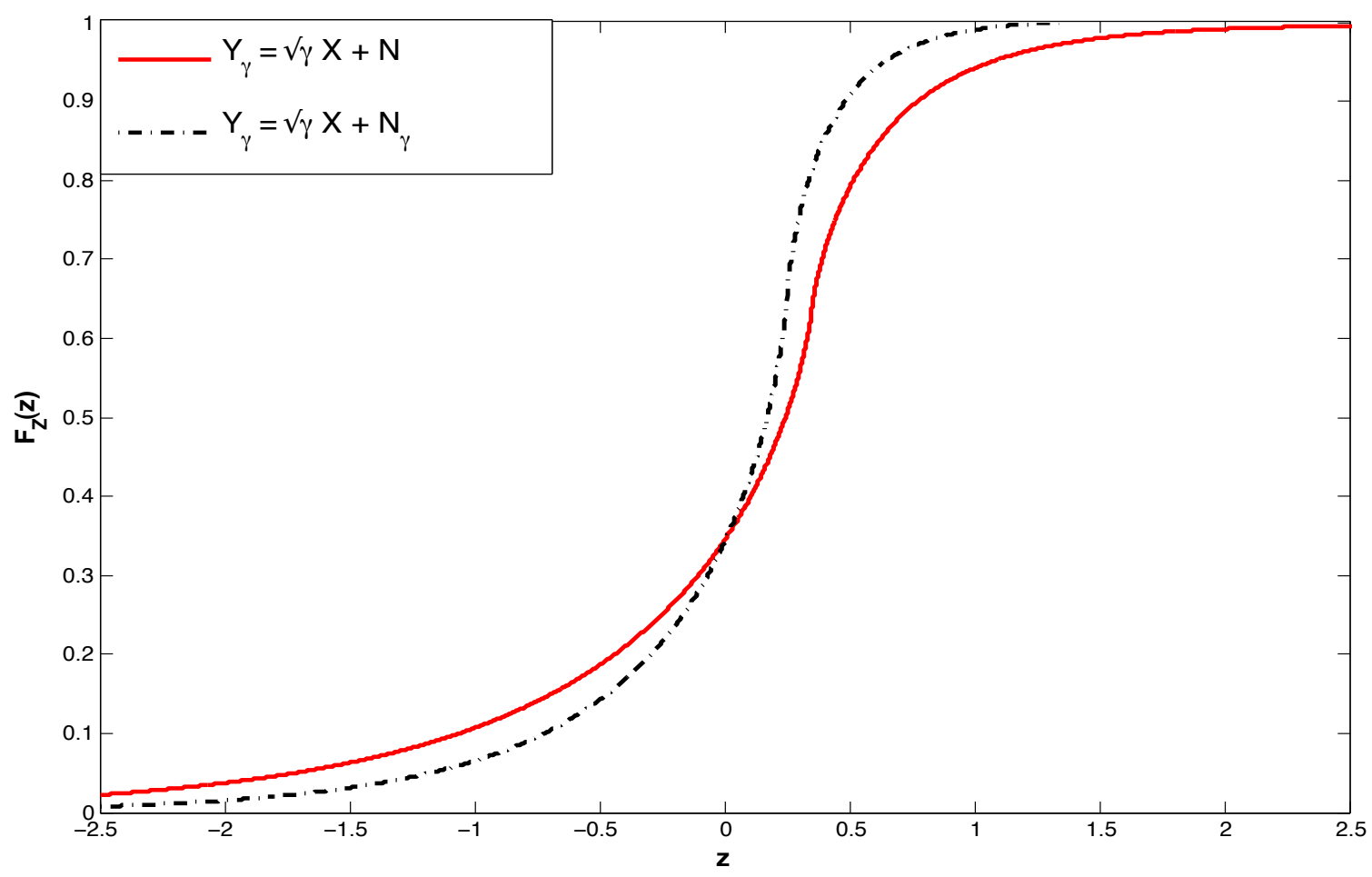

Fig. 1: C.D.F. of $Z$ under the couplings discussed in Subsections III-B and III-C for a Gaussian input

We now evaluate $I_{2}$ as defined in 70 for this process

$$
\begin{aligned}
I_{2} & =\frac{1}{2} \int_{0}^{\mathrm{snr}}\left(X-\mathrm{E}\left[X \mid Y_{\gamma}\right]\right)^{2} d \gamma \\
& =\sum_{i=1}^{M} \int_{(i-1) \Delta}^{i \Delta}\left(X-\mathrm{E}\left[X \mid Y_{\gamma}\right]\right)^{2} d \gamma
\end{aligned}
$$

We are interested in the limiting process when $\Delta$ is small. We consider the Riemann sum approximation of the above integral,

$$
\begin{aligned}
S_{M} & =\sum_{i=1}^{M}\left(X-\mathrm{E}\left[X \mid Y_{i \Delta}\right]\right)^{2} \Delta \\
& =\frac{\operatorname{snr}}{M} \sum_{i=1}^{M}\left(X-\mathrm{E}\left[X \mid Y_{i \Delta}\right]\right)^{2}
\end{aligned}
$$

We now look at the conditional variance of $I_{2}$ given $X$

$$
\operatorname{Var}\left[I_{2} \mid X\right]=\frac{\mathrm{snr}^{2}}{M^{2}} \sum_{i=1}^{M} \operatorname{Var}\left[\left(X-\mathrm{E}\left[X \mid Y_{i \Delta}\right]\right)^{2} \mid X\right],
$$

where $\operatorname{Var}[\cdot \mid X]$ denotes the conditional variance averaged over the distribution of $X$. Thus, under mild regularity conditions on the underlying distribution of $X$ (for instance, $\mathrm{E}\left[X^{4}\right]<\infty$ suffices) we can see that the summand 
in the r.h.s. is bounded above by a constant independent of $M$. Therefore the sum involves adding over $M$ terms that are $O(1)$. In other words

$$
\operatorname{Var}\left[I_{2} \mid X\right]=\frac{O(M)}{M^{2}} \quad \text { in probability }
$$

and thus

$$
\text { l.i.m. } \text {. }_{\rightarrow \infty} I_{2}=\mathrm{E}\left[I_{2} \mid X\right]
$$

Therefore, $Z=I_{1}-I_{2}$, the tracking error is given (in the li.i.m. sense as $M \rightarrow \infty$ ) by

$$
\begin{aligned}
Z & =I_{1}-I_{2} \\
& =I_{1}-\mathrm{E}\left[I_{2} \mid X\right] \\
& =I_{1}-\mathrm{E}\left[I_{1} \mid X\right]
\end{aligned}
$$

where the last equality follows from equation $(77)$. We now consider the case when $X \sim \mathcal{N}(0,1)$ which satisfies (91), and thus we can apply (94) to explicitly calculate $Z$.

Note that $I_{1}\left(X, Y_{\mathrm{snr}}\right)$, as defined in Definition 1 , depends only on the joint distribution of $X$ and $Y_{\text {snr }}$, and is therefore the same for all the channel couplings that are consistent with (76), for a fixed input distribution on $X$. Thus, for $X \sim \mathcal{N}(0,1), I_{1}$ can be computed explicitly using Definition 1 to yield,

$$
I_{1}=\frac{1}{2} \log (1+\mathrm{snr})+\frac{1}{2}\left(\frac{Y^{2}}{1+\mathrm{snr}}-(Y-\sqrt{\operatorname{snr}} X)^{2}\right)
$$

and

$$
\mathrm{E}\left[I_{1} \mid X\right]=\frac{1}{2} \log (1+\mathrm{snr})+\frac{1}{2}\left(\frac{\mathrm{snr}}{1+\mathrm{snr}}\left(X^{2}-1\right)\right) .
$$

Let $N=Y_{\mathrm{snr}}-\sqrt{\mathrm{snr}} X$. Using 95 and 96 , we simplify 94 to get the following closed form expression for $Z$ :

$$
Z=\frac{1}{2(1+\mathrm{snr})}\left(-N^{2} \mathrm{snr}+2 X N \sqrt{\mathrm{snr}}+\mathrm{snr}\right) .
$$

Further, the variance is given by,

$$
\operatorname{Var}(Z)=\operatorname{snr} \frac{1+2 \mathrm{snr}}{2(1+\mathrm{snr})^{2}}
$$

For snr=1, we present a plot of the Cumulative Distribution Function of the random variable $Z$ in 94 in Figure 1 .

\section{Comparison of Variances}

Previously, in III-A, III-B and III-C we have considered different couplings that are consistent with (76) and give rise to different pointwise relations between $X$ and $Y_{0}^{\mathrm{snr}}$. In particular, for the specific channel input $X \sim \mathcal{N}(0,1)$, we have explicit characterizations of the tracking error $Z$ defined in Definition 1 . We have also calculated the variance of this tracking error for each of these process evolutions, and they are given by (75, 82, and (98) respectively. Here, we compare these couplings in terms of the variance of the tracking error for the Gaussian 


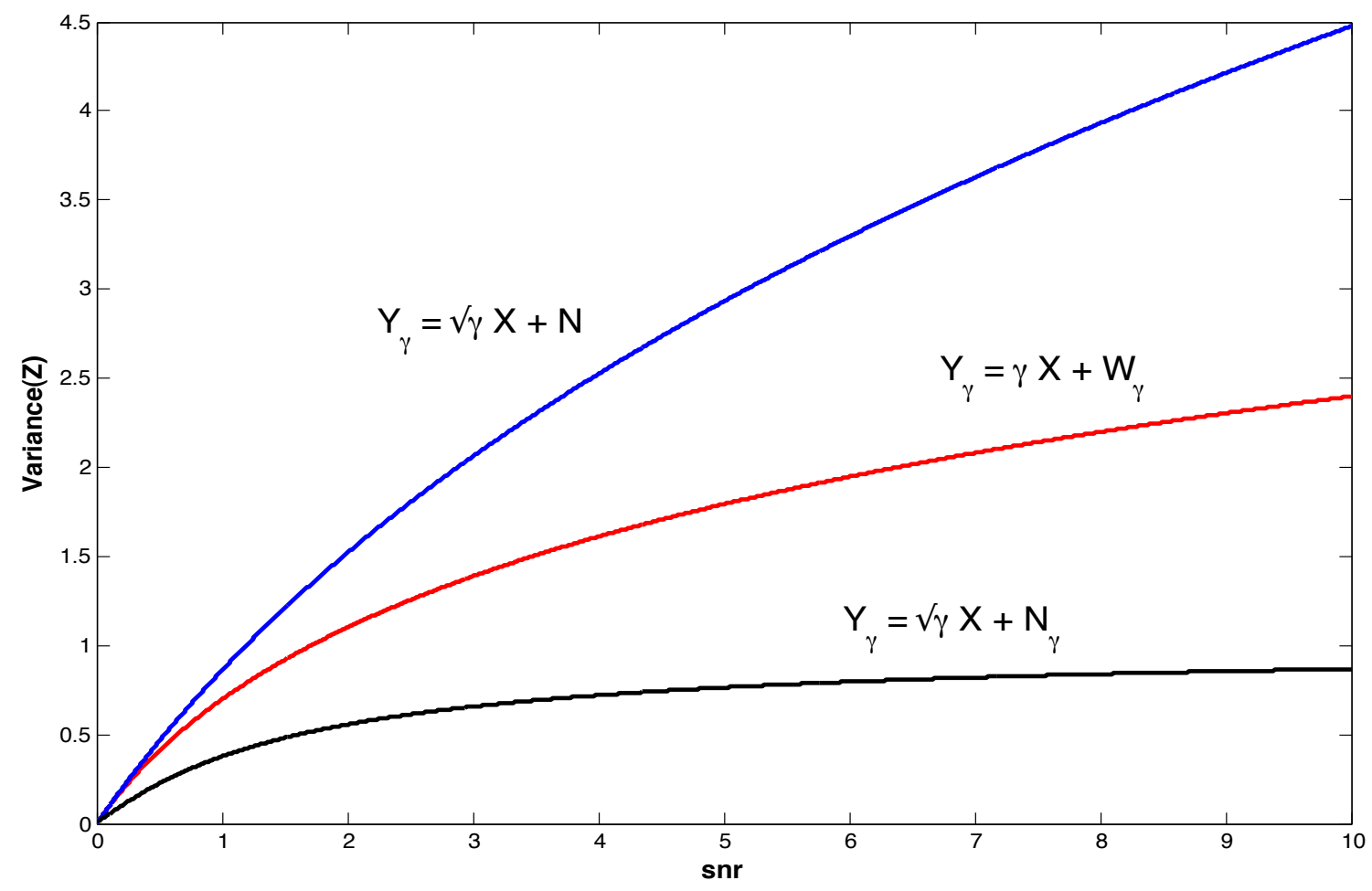

Fig. 2: Variance of Tracking Error $Z$ vs. snr for the cases in sections III-A III-B and III-C

input. This comparison effectively tells us which particular relationship results in a better pointwise tracking of the information density and the actual squared error of the MMSE estimators. Fig. 2 shows a plot of the error variances. We observe that in this example of a Gaussian input, the coupling $\Pi$ III-C results in the lowest variance of the tracking error, while that of III-B in the highest variance. We conjecture that to be the case in general, i.e., for any distribution of $X$ with finite power.

Remark 2: Similar to our presentation of alternative couplings in the scalar estimation problem, we could introduce a time-snr coupling of inputs and outputs in the continuous time estimation model different from the Brownian sheet dependence in Section [II-B4 The difference in behavior observed would be consistent with the differences we observe in the scalar scenario. However, a detailed analysis of the continuous time I-MMSE relationship for processes under alternative couplings is beyond the scope of this paper.

\section{E. An identity}

As a final result, we present an interesting identity between two random quantities. The nature and applicability of such an identity needs to be explored further, but at the very least it shows us the kind of identities that can 
easily be reaped from our pointwise framework. Let $X \sim P$. We consider two different channels as follows:

$$
\begin{aligned}
& Y_{\gamma}=\sqrt{\gamma} X+W_{1} \\
& \tilde{Y}_{\gamma}=\gamma X+W_{\gamma},
\end{aligned}
$$

where $W_{\gamma}$ is a standard Brownian motion, independent of $X$. Thus, the coupling in 99 is the "Additive Standard Gaussian" one of Subsection III-B (with the role of $N$ played by $W_{1}$ ), while that in 100 is our original Brownian motion coupling. We note that

$$
Y_{1}=\tilde{Y}_{1}
$$

and consequently,

$$
\log \frac{d P_{Y_{1} \mid X}}{d P_{Y_{1}}}=\log \frac{d P_{\tilde{Y}_{1} \mid X}}{d P_{\tilde{Y}_{1}}} \quad \text { a.s. }
$$

However, we are now in a position to invoke results derived in Section II-A1 to characterize the quantities on either side of (102). Using the definition of $Z$ in Definition 1 and Proposition 1 , we get respectively

$$
\begin{aligned}
& Z_{1}=\log \frac{d P_{Y_{1} \mid X}}{d P_{Y_{1}}}-\frac{1}{2} \int_{0}^{1}\left(X-\mathrm{E}\left[X \mid Y_{\gamma}\right]\right)^{2} d \gamma \\
& \tilde{Z}_{1}=\log \frac{d P_{\tilde{Y}_{1} \mid X}}{d P_{\tilde{Y}_{1}}}-\frac{1}{2} \int_{0}^{1}\left(X-\mathrm{E}\left[X \mid \tilde{Y}_{\gamma}\right]\right)^{2} d \gamma=\int_{0}^{1}\left(X-\mathrm{E}\left[X \mid \tilde{Y}_{\gamma}\right]\right) d W_{\gamma} \quad \text { a.s. }
\end{aligned}
$$

Combining the above we get

$$
\begin{aligned}
Z_{1} & =\log \frac{d P_{Y_{1} \mid X}}{d P_{Y_{1}}}-\frac{1}{2} \int_{0}^{1}\left(X-\mathrm{E}\left[X \mid Y_{\gamma}\right]\right)^{2} d \gamma \\
& =\log \frac{d P_{\tilde{Y}_{1} \mid X}}{d P_{\tilde{Y}_{1}}}-\frac{1}{2} \int_{0}^{1}\left(X-\mathrm{E}\left[X \mid Y_{\gamma}\right]\right)^{2} d \gamma \quad \text { a.s. } \\
& =\int_{0}^{1}\left(X-\mathrm{E}\left[X \mid \tilde{Y}_{\gamma}\right] d W_{\gamma}+\frac{1}{2} \int_{0}^{1}\left(X-\mathrm{E}\left[X \mid \tilde{Y}_{\gamma}\right]\right)^{2} d \gamma-\frac{1}{2} \int_{0}^{1}\left(X-\mathrm{E}\left[X \mid Y_{\gamma}\right]\right)^{2} d \gamma \quad\right. \text { a.s. }
\end{aligned}
$$

where all equalities are valid in the almost sure sense. Note that the relation in (107) is not only consistent with (and immediately implies) the I-MMSE relation, but also exhibits the pointwise relation between two different channel couplings. We can further plug in the expressions we have to characterize $Z_{1}$ (in subsection III-B], to get equality relationships coupling the two channels considered.

\section{PROOFS}

Having discussed the main results in Section II] we now present the proofs in detail. We begin by proving our main result for the "Pointwise Duncan" setting in Proposition 3 . We then note that for the special case when the input is a DC process, the scalar estimation result in Proposition 1 follows directly from the continuous-time result.

Proof of Proposition 3 . We recall that the input-output mutual information is the expected value of the $\log$ Radon-Nikodym derivative of the measure induced by the process $\left\{Y^{T}\right\}$ conditioned on $X^{T}$ with respect to the measure induced by the process $\left\{Y^{T}\right\}$. The expectation is with respect to the law $P$. 
Define the causal estimate of $X_{t}$,

$$
\hat{X}_{t}=\mathrm{E}\left[X_{t} \mid Y_{0}^{t}\right]
$$

We characterize the information density, by introducing the Radon-Nikodym derivative with respect to the standard Wiener measure [11]. Using the definition, and taking logarithm we get

$$
\begin{aligned}
\log \frac{d P_{Y^{T} \mid X^{T}}}{d P_{Y^{T}}} & =\log \left(\frac{d P_{Y^{T} \mid X^{T}}}{d \mu} / \frac{d P_{Y^{T}}}{d \mu}\right) \\
& =\log \frac{d P_{Y^{T} \mid X^{T}}}{d \mu}-\log \frac{d P_{Y^{T}}}{d \mu} .
\end{aligned}
$$

Let $\mu$ denote the standard Wiener measure on $Y^{T}$. We apply the Girsanov theorem [12] to denote the RadonNikodym derivatives of the conditional and marginal laws of $Y^{T} \mid X^{T}$ and $Y^{T}$ respectively with respect to $\mu$, as follows:

$$
\begin{aligned}
\log \frac{d P_{Y^{T} \mid X^{T}}}{d \mu} & =\int_{0}^{T} X_{t} d Y_{t}-\frac{1}{2} \int_{0}^{T} X_{t}^{2} d t \\
\log \frac{d P_{Y^{T}}}{d \mu} & =\int_{0}^{T} \hat{X}_{t} d Y_{t}-\frac{1}{2} \int_{0}^{T}\left(\hat{X}_{t}\right)^{2} d t
\end{aligned}
$$

where the equalities hold almost surely (a.s.). Therefore, from (110), (111) and (112) we have

$$
\log \frac{d P_{Y^{T} \mid X^{T}}}{d P_{Y^{T}}}=\int_{0}^{T}\left(X_{t}-\hat{X}_{t}\right) d Y_{t}-\frac{1}{2} \int_{0}^{T}\left(X_{t}^{2}-\left(\hat{X}_{t}\right)^{2}\right) d t .
$$

We shall now proceed to simplify (113). Note from [19), (snr=1)

$$
\begin{aligned}
\log \frac{d P_{Y^{T} \mid X^{T}}}{d P_{Y^{T}}} & =\int_{0}^{T}\left(X_{t}-\hat{X}_{t}\right)\left(X_{t} d t+d W_{t}\right)-\frac{1}{2} \int_{0}^{T}\left(X_{t}^{2}-\left(\hat{X}_{t}\right)^{2}\right) d t \\
& =\int_{0}^{T}\left(X_{t}^{2}-X_{t} \hat{X}_{t}-\frac{1}{2} X_{t}^{2}+\frac{1}{2}\left(\hat{X}_{t}\right)^{2}\right) d t+\int_{0}^{T}\left(X_{t}-\hat{X}_{t}\right) d W_{t} \\
& =\frac{1}{2} \int_{0}^{T}\left(X_{t}-\hat{X}_{t}\right)^{2} d t+\int_{0}^{T}\left(X_{t}-\hat{X}_{t}\right) d W_{t} .
\end{aligned}
$$

On re-arranging, we get the desired result

$$
D(T)=\int_{0}^{T}\left(X_{t}-\mathrm{E}\left[X_{t} \mid Y_{0}^{t}\right]\right) d W_{t} \quad \text { a.s. }
$$

It is instructive to note that for the special case when $X_{t} \equiv X$ and $T=\operatorname{snr}$, Proposition 3 reduces directly to the scalar estimation setting in Section [I-A1 thereby giving a direct proof of Proposition 1 . We now note that Theorem 3 also follows directly from Proposition 3 , by using a familiar identity for Itô integrals. 
Proof of Theorem 3 .

$$
\begin{aligned}
\operatorname{Var}(D(T)) & =\mathrm{E}\left[\{D(T)\}^{2}\right] \\
& =\mathrm{E}\left[\left\{\int_{0}^{T}\left(X_{t}-E\left[X_{t} \mid Y^{t}\right]\right) d W_{t}\right\}^{2}\right] \\
& =\int_{0}^{T} E\left[\left(X_{t}-E\left[X_{t} \mid Y^{t}\right]\right)^{2}\right] d t . \\
& =\mathrm{cmmse}(T)=2 I\left(X^{T} ; Y^{T}\right)
\end{aligned}
$$

Here, 118 follows from the fact that $\mathrm{E}[D(T)]=0$, and $[120$ is a consequence of Itô's Isometry property [13. Chapter 6]. Note also, that by definition, [120] is the squared filtering error, denoted by cmmse(T) in [20], which in turn is twice the mutual information by Duncan's theorem.

We again observe that for the special choices $X_{t} \equiv X$ and $T=$ snr, we obtain the result for the scalar setting in Theorem 1, which tells us that the variance of the tracking error is equal to the minimum mean squared error integrated over SNR. Having established the pointwise results for the scalar and continuous time channels using Girsanov theory, we now proceed to prove the limit theorem that is a direct application of the pointwise treatment of Duncan's result.

Proof of Theorem 4 Note that Duncan's theorem tells us the equivalence of mutual information rate and half time-averaged causal squared error, i.e.

$$
\frac{1}{T} I\left(X^{T} ; Y^{T}\right)=\frac{1}{T} \int_{0}^{T} \frac{1}{2} \mathrm{E}\left[\left(X_{t}-E\left[X_{t} \mid Y_{0}^{t}\right]\right)^{2}\right] d t .
$$

Under the channel model in (19), we can change the order of expectation to equivalently state the above identity as:

$$
\mathrm{E}\left[\frac{1}{T} \log \frac{d P_{Y_{0}^{T} \mid X_{0}^{T}}}{d P_{Y_{0}^{T}}}\right]=\mathrm{E}\left[\frac{1}{T} \int_{0}^{T} \frac{1}{2}\left(X_{t}-\mathrm{E}\left[X_{t} \mid Y_{0}^{t}\right]\right)^{2} d t\right] .
$$

Thus we observe the equality in expectation of the information density and half the squared filtering error. Using our pointwise characterization of Duncan's result, we will now show that not only are the random quantities in 123) equal in expectation, but in the limit of large $\mathrm{T}$, their difference converges to 0 in the mean square sense. Let us recall (22) and the result in (24). On dividing both sides of (24) by $T$, we get the following:

$$
\frac{1}{T} \log \frac{d P_{Y_{0}^{T} \mid X_{0}^{T}}}{d P_{Y_{0}^{T}}}=\frac{1}{T} \int_{0}^{T} \frac{1}{2}\left(X_{t}-\mathrm{E}\left[X_{t} \mid Y_{0}^{t}\right]\right)^{2} d t+\frac{1}{T} \int_{0}^{T}\left(X_{t}-E\left[X_{t} \mid Y_{0}^{t}\right]\right) \cdot d W_{t} .
$$

Let us take the limit as $T \rightarrow \infty$. We claim that under very basic regularity conditions on $X_{t}$, the second term in the r.h.s. goes to 0 in the mean square sense.

$$
\begin{aligned}
\operatorname{Var}\left(\frac{1}{T} \int_{0}^{T}\left(X_{t}-E\left[X_{t} \mid Y_{0}^{t}\right]\right) \cdot d W_{t}\right) & =\frac{1}{T^{2}} \operatorname{Var}\left(\int_{0}^{T}\left(X_{t}-E\left[X_{t} \mid Y_{0}^{t}\right]\right) \cdot d W_{t}\right) \\
& =\frac{1}{T^{2}} \int_{0}^{T} E\left[\left(X_{t}-E\left[X_{t} \mid Y^{t}\right]\right)^{2}\right] d t
\end{aligned}
$$


Thus, if the expression in 126 goes to 0 in the limit $T \rightarrow \infty$, i.e. it satisfies the condition in 27], then from (124) we get the desired result:

$$
\text { l.i.m. } T \rightarrow \infty \frac{1}{T}\left[\log \frac{d P_{Y_{0}^{T} \mid X_{0}^{T}}}{d P_{Y_{0}^{T}}}-\frac{1}{2} \int_{0}^{T}\left(X_{t}-\mathrm{E}\left[X_{t} \mid Y_{0}^{t}\right]\right)^{2} d t\right]=0,
$$

where 1.i.m. denotes Limit in the Mean.

Having established the pointwise representation of Duncan's result and using it to extract results for scalar estimation, we now present the proof for continuous-time estimation with mismatch. We again note in this case, that the scalar estimation results follow as a special case of the continuous-time version of the result.

Proof of Proposition 4: Let us denote the causal estimates of $X_{t}$ under each law:

$$
\begin{aligned}
\pi_{t}^{P} & =E_{P}\left[X_{t} \mid Y^{t}\right] \\
\pi_{t}^{Q} & =E_{Q}\left[X_{t} \mid Y^{t}\right] .
\end{aligned}
$$

We first note that the innovations processes specified below,

$$
\bar{W}_{t}=Y_{t}-\int_{0}^{t} \pi_{s}^{P} d s
$$

and

$$
\bar{V}_{t}=Y_{t}-\int_{0}^{t} \pi_{s}^{Q} d s
$$

are standard Brownian motions under $P$ and $Q$. We now apply Girsanov's theorem (also discussed in [4. Section IV.D]) to characterize the $\log$ Radon-Nikodym derivative of the observed process under laws $P$ and $Q$ respectively,

$$
\begin{aligned}
\log \frac{d P_{Y^{T}}}{d \mu} & =\int_{0}^{T} \pi_{t}^{P} d Y_{t}-\frac{1}{2} \int_{0}^{T}\left(\pi_{t}^{P}\right)^{2} d t \\
\log \frac{d Q_{Y^{T}}}{d \mu} & =\int_{0}^{T} \pi_{t}^{Q} d Y_{t}-\frac{1}{2} \int_{0}^{T}\left(\pi_{t}^{Q}\right)^{2} d t .
\end{aligned}
$$

We now use 132 and (133) to characterize the log Radon-Nikodym derivative of the measure induced by the output process $Y_{0}^{T}$ under $P$ with respect to the measure induced under $Q$,

$$
\begin{aligned}
\log \frac{d P_{Y_{0}^{T}}}{d Q_{Y_{0}^{T}}} & =\log \left(\frac{d P_{Y_{0}^{T}} / d \mu}{d Q_{Y_{0}^{T}} / d \mu}\right) \\
& =\int_{0}^{T}\left(\pi_{t}^{P}-\pi_{t}^{Q}\right) d Y_{t}-\frac{1}{2} \int_{0}^{T}\left[\left(\pi_{t}^{P}\right)^{2}-\left(\pi_{t}^{Q}\right)^{2}\right] d t \\
& =\int_{0}^{T}\left(\pi_{t}^{P}-\pi_{t}^{Q}\right)\left(X_{t} d t+d W_{t}\right)-\frac{1}{2} \int_{0}^{T}\left[\left(\pi_{t}^{P}\right)^{2}-\left(\pi_{t}^{Q}\right)^{2}\right] d t \\
& =\frac{1}{2} \int_{0}^{T}\left[-\left(\pi_{t}^{P}\right)^{2}+2 \pi_{t}^{P} X_{t}-2 \pi_{t}^{Q} X_{t}+\left(\pi_{t}^{Q}\right)^{2}\right] d t+\int_{0}^{T}\left(\pi_{t}^{P}-\pi_{t}^{Q}\right) d W_{t} \\
& =\frac{1}{2} \int_{0}^{T}\left[\left(\pi_{t}^{Q}-X_{t}\right)^{2}-\left(\pi_{t}^{P}-X_{t}\right)^{2}\right] d t+\int_{0}^{T}\left(\pi_{t}^{P}-\pi_{t}^{Q}\right) d W_{t},
\end{aligned}
$$

where 136 follows from (19). 
Note from 31 and 138 that, $M(T)$ is a stochastic integral and is expressed as:

$$
M(T)=\int_{0}^{T}\left(\pi_{t}^{P}-\pi_{t}^{Q}\right) d W_{t} \quad P-a . s .
$$

We now invoke Itô's Isometry property along with Proposition 4 to prove the second moment result in Theorem 5 .

Proof of Theorem 5 . Using the isometry property of Itô integrals, we can compute the variance of $M(T)$ as follows

$$
\begin{aligned}
\operatorname{Var}(M(T)) & =E_{P}\left[\int_{0}^{T}\left(\pi_{t}^{P}-\pi_{t}^{Q}\right)^{2} d t\right] \\
& =\int_{0}^{T} E_{P}\left[\left(\pi_{t}^{Q}-X_{t}\right)^{2}-\left(\pi_{t}^{P}-X_{t}\right)^{2}\right] d t \\
& =\operatorname{cmse}_{P, Q}(T)-\text { cmse }_{P, P}(T), \\
& =2 D\left(P_{Y_{0}^{T}} \| Q_{Y_{0}^{T}}\right)
\end{aligned}
$$

where (141) follows from orthogonality property of estimators, and (143) follows from 30.

We now present the proofs of the pointwise results generalized to channels with feedback. The techniques are similar to the ones we use in order to prove the previous results for pointwise Duncan and mismatch.

Proof of Theorem 6. In proving the pointwise result for channels with feedback, we use the same idea that we employed in the pointwise treatment of Duncan's theorem. We use Girsanov theory to characterize the likelihood ratio of the conditional and marginal laws of $Y^{T}$, in terms of the filtering error in estimating $\phi_{t}$. Let $\mu$ be the standard Wiener measure. Then,

$$
\begin{aligned}
\log \frac{d P_{Y^{T} \mid X^{T}}}{d P_{Y^{T}}} & =\log \left(\frac{d P_{Y^{T} \mid X^{T}}}{d \mu} / \frac{d P_{Y^{T}}}{d \mu}\right) \\
& =\log \frac{d P_{Y^{T} \mid X^{T}}}{d \mu}-\log \frac{d P_{Y^{T}}}{d \mu} \quad \text { a.s. }
\end{aligned}
$$

Using the formula for likelihood ratios in the presence of white noise (cf. [5],[11], [14]), we get the following almost-sure characterizations for the log Radon-Nikodym derivatives

$$
\begin{aligned}
\log \frac{d P_{Y^{T} \mid X^{T}}}{d \mu} & =\int_{0}^{T} \phi_{t} d Y_{t}-\frac{1}{2} \int_{0}^{T} \phi_{t}^{2} d t \\
\log \frac{d P_{Y^{T}}}{d \mu} & =\int_{0}^{T} \hat{\phi}_{t} d Y_{t}-\frac{1}{2} \int_{0}^{T}\left(\hat{\phi}_{t}\right)^{2} d t,
\end{aligned}
$$

where

$$
\hat{\phi}_{t}=\mathrm{E}\left[\phi_{t} \mid Y_{0}^{t}\right]
$$

is the causal estimate of $\phi_{t}$ given the observations up until $t$. From equations (145), 146, and 147), we get that

$$
\log \frac{d P_{Y^{T} \mid X^{T}}}{d P_{Y^{T}}}=\int_{0}^{T}\left(\phi_{t}-\hat{\phi}_{t}\right)\left(d Y_{t}\right)-\frac{1}{2} \int_{0}^{T}\left(\phi_{t}^{2}-\left(\hat{\phi}_{t}\right)^{2}\right) d t \quad \text { a.s. }
$$


We recall that

$$
d Y_{t}=\phi_{t} d t+d W_{t},
$$

and simplify 149$)$ to get

$$
\log \frac{d P_{Y^{T} \mid X^{T}}}{d P_{Y^{T}}}=\frac{1}{2} \int_{0}^{T}\left(\phi_{t}-\hat{\phi}_{t}\right)^{2} d t+\int_{0}^{T}\left(\phi_{t}-\hat{\phi}_{t}\right) d W_{t} \quad \text { a.s. }
$$

Recall that

$$
D_{\phi}(T) \triangleq \log \frac{d P_{Y^{T} \mid X^{T}}}{d P_{Y^{T}}}-\frac{1}{2} \int_{0}^{T}\left(\phi_{t}-\hat{\phi}_{t}\right)^{2} d t
$$

Combining with (151), we get the desired result,

$$
D_{\phi}(T)=\int_{0}^{T}\left(\phi_{t}-\hat{\phi}_{t}\right) d W_{t} \quad \text { a.s. }
$$

We now use Theorem 6 along with Itô's Isometry to directly yield the result in Corollary 7

$$
\begin{aligned}
\operatorname{Var}\left(D_{\phi}(T)\right) & =\mathrm{E}\left[\left(\int_{0}^{T}\left(\phi_{t}-\hat{\phi}_{t}\right) d W_{t}\right)^{2}\right] \\
& \stackrel{(a)}{=} \int_{0}^{T} \mathrm{E}\left[\left(\phi_{t}-\hat{\phi}_{t}\right)^{2}\right] d t \\
& =\operatorname{cmmse}_{\phi}(T),
\end{aligned}
$$

where (a) follows from the Isometry property of stochastic integrals.

Note: Theorem 8 and Corollary 9 can be proved using the same techniques we used to establish the previous results for the non-mismatched case in Theorem 6, and Proposition 4 for the mismatched setting without feedback. Since the proofs follow directly, we omit them from the present discussion.

We now explore the proof of the pointwise I-MMSE relationship for processes. We recall that in this setting, we have used a Brownian sheet process [52] to place the input and output processes on the same probability space on the time-snr plane. In this proof we use higher dimensional Girsanov theory to characterize the Radon-Nikodym derivative for vector processes. The proof is given below.

\section{Proof of Theorem 10 .}

We first present a result which can be established using Girsanov theory for higher dimensions for the continuous time Gaussian channel.

Let $\mathbf{X} \in \mathbb{R}^{M}$ be a real-valued random vector governed by the law $P_{\mathbf{X}}$. This acts as input to a Gaussian channel (at SNR = 1) to yield the output $\mathbf{Y}_{t}$ as a function of observation time $t \in[0, T]$. The input and output are related by the following equation:

$$
\mathbf{Y}_{t}=t \mathbf{X}+\mathbf{W}_{t} \quad t \in[0, \mathrm{snr}]
$$

where $\mathbf{W}_{t}$ is a standard Brownian motion in $M$ dimensions. In this setting we are interested in the "tracking error" between the log Radon-Nikodym derivative and integral of half the squared error, in the time interval $[0$, snr $]$. The 
corresponding definitions for the vector case are listed below:

$$
\begin{aligned}
I_{1} & =\log \frac{d P_{\mathbf{Y}_{\mathrm{snr}}} \mathbf{X}}{d P_{\mathbf{Y}_{\mathrm{snr}}}} \\
I_{2} & =\frac{1}{2} \int_{0}^{\mathrm{snr}}\left\|\mathbf{X}-\mathrm{E}\left[\mathbf{X} \mid \mathbf{Y}_{0}^{t}\right]\right\|^{2} d t
\end{aligned}
$$

Lemma 12: Let $\mathbf{Z}=I_{1}-I_{2}$, where $I_{1}$ and $I_{2}$ are defined in [158)-(159). Then,

$$
\mathbf{Z}=\int_{0}^{\mathrm{snr}}\left(\mathbf{X}-\mathrm{E}\left[\mathbf{X} \mid \mathbf{Y}_{0}^{t}\right) \cdot d \mathbf{W}_{t}, \quad\right. \text { a.s. }
$$

Proof: The proof is very similar to that of Theorem 3 Let $\mu$ be the standard Wiener measure. We note that

$$
\begin{aligned}
I_{1} & =\log \frac{d P_{\mathbf{Y}_{\mathrm{snr}} \mid \mathbf{X}}}{d P_{\mathbf{Y}_{\mathrm{snr}}}} \\
& =\log \frac{d P_{\mathbf{Y}_{\mathrm{snr}} \mid \mathbf{X}}}{d \mu}-\log \frac{d P_{\mathbf{Y}_{\mathrm{snr}}}}{d \mu} \quad \text { a.s. }
\end{aligned}
$$

Define

$$
\hat{\mathbf{X}}_{\gamma}=\mathrm{E}\left[\mathbf{X} \mid \mathbf{Y}_{0}^{\gamma}\right]
$$

for $\gamma \in[0$, snr $]$. Now, using Girsanov theorem (cf. [7, Section 3.5]) for higher dimensions, we can write the $\log$ Radon-Nikodym derivatives of the conditional and marginal distributions of $\mathbf{Y}$, with respect to $\mu$ as follows,

$$
\begin{aligned}
\log \frac{d P_{\mathbf{Y}_{\mathrm{snr}} \mid \mathbf{X}}}{d \mu} & =\int_{0}^{\mathrm{snr}} \mathbf{X} \cdot d \mathbf{Y}_{\gamma}-\frac{1}{2} \int_{0}^{\mathrm{snr}} \mathbf{X} \cdot \mathbf{X} d \gamma \quad \text { a.s. } \\
\log \frac{d P_{\mathbf{Y}_{\mathrm{snr}}}}{d \mu} & =\int_{0}^{\mathrm{snr}} \hat{\mathbf{X}}_{\gamma} \cdot d \mathbf{Y}_{\gamma}-\frac{1}{2} \int_{0}^{\mathrm{snr}} \hat{\mathbf{X}}_{\gamma} \cdot \hat{\mathbf{X}}_{\gamma} d \gamma \quad \text { a.s. }
\end{aligned}
$$

Combining the above expressions with (162) and using (157), we get

$$
I_{1}=\int_{0}^{\mathrm{snr}}\left(\mathbf{X}-\hat{\mathbf{X}}_{\gamma}\right) \cdot d \mathbf{Y}_{\gamma}-\frac{1}{2} \int_{0}^{\mathrm{snr}}\left(\mathbf{X} \cdot \mathbf{X}-\hat{\mathbf{X}}_{\gamma} \cdot \hat{\mathbf{X}}_{\gamma}\right) d \gamma
$$

which upon simplification reduces to,

$$
I_{1}=\frac{1}{2} \int_{0}^{\mathrm{snr}}\left\|\mathbf{X}-\hat{\mathbf{X}}_{\gamma}\right\|^{2} d \gamma+\int_{0}^{\mathrm{snr}}\left(\mathbf{X}-\hat{\mathbf{X}}_{\gamma}\right) \cdot d \mathbf{W}_{\gamma} \quad \text { a.s. }
$$

In other words,

$$
\begin{aligned}
\mathbf{Z} & =I_{1}-I_{2} \\
& =\int_{0}^{\mathrm{snr}}\left(\mathbf{X}-\hat{\mathbf{X}}_{\gamma}\right) \cdot d \mathbf{W}_{\gamma} \quad \text { a.s. }
\end{aligned}
$$

Armed with this characterization in 160 , of the input-output information density in higher dimensions for the Gaussian channel in [52, we now proceed to establish a relationship akin to the I-MMSE for a specific class of processes. We begin by looking at piecewise constant scalar processes. The result we obtain (we shall argue), can be extended just as easily to the general class of finite power continuous-time processes using approximation arguments. 
Let $\tilde{X}_{t}$ be a continuous time process, and $\tilde{Y}_{t}$ be its noise corrupted version through the channel in 19 ) observed in the interval $[0, T]$. Fix $M \in \mathbb{N}$. We let $\tilde{X}_{t}$ be a piecewise-constant process such that

$$
\tilde{X}_{t} \equiv X_{i} \quad \text { for all } \frac{(i-1)}{M} T \leq t \leq \frac{i}{M} T
$$

where $X_{i} \sim P$, and $1 \leq i \leq M$.

Fix snr $>0$. From [2] we know that the mutual information is the integral over SNR of the smoothing error,

$$
I\left(\tilde{X}_{0}^{T} ; \tilde{Y}_{0}^{T,(\mathrm{snr})}\right)=\frac{1}{2} \int_{0}^{\mathrm{snr}}\left(\tilde{X}_{t}-\mathrm{E}\left[\tilde{X}_{t} \mid \tilde{Y}_{0}^{T,(\gamma)}\right]\right)^{2} d \gamma
$$

Note that,

$$
\mathrm{E}\left[\log \frac{d P_{\tilde{Y}_{0}^{T,(\mathrm{snr})} \mid \tilde{X}_{0}^{T}}}{d P_{\tilde{Y}_{0}^{T,(\mathrm{srr})}}}\right]=I\left(\tilde{X}_{0}^{T} ; \tilde{Y}_{0}^{T,(\mathrm{snr})}\right)=I\left(\left\{X_{i}\right\}_{i=1}^{M} ;\left\{Y_{\mathrm{snr}}^{[i]}\right\}_{i=1}^{M}\right)=I\left(\mathbf{X} ; \mathbf{Y}_{\mathrm{snr}}\right)=\mathrm{E}\left[\log \frac{\left.d P_{\mathbf{Y}_{\mathrm{snr}} \mid \mathbf{X}}\right]}{d P_{\mathbf{Y}_{\mathrm{snr}}}}\right]
$$

where

$$
Y_{\gamma}^{[i]}=\gamma \frac{T}{M} X_{i}+W_{\gamma}^{[i]}
$$

and $\gamma \in[0, \mathrm{snr}]$ denotes the signal to noise ratio.

- The random vectors $\mathbf{X} \in \mathbb{R}^{M}$ and $\mathbf{Y}_{\gamma} \in \mathbb{R}^{M}$ denote the collection of variables $\left\{X_{i}\right\}_{i=1}^{M}$ and $\left\{Y_{\gamma}^{[i]}\right\}_{i=1}^{M}$ respectively.

- $W_{\gamma}^{[i]}$ denotes the Brownian sheet process $W_{t}^{(\gamma)}$ (that drives the channel noise in 52), observed in the interval $\left[\frac{(i-1)}{M} T, \frac{i}{M} T\right]$. The M-dimensional random vector $\mathbf{W}_{\gamma}=\left\{W_{\gamma}^{[i]}\right\}_{i=1}^{M}$ denotes an M-dimensional Brownian motion indexed by $\gamma$.

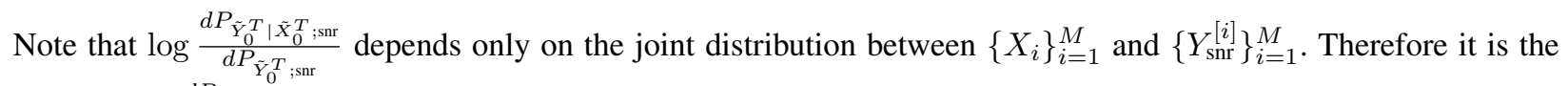
same as $\log \frac{d P_{\mathbf{Y}_{\text {snr }}} \mathbf{X}}{d \mathbf{P}_{\mathbf{S n r}}}$, not only under expectation, but also pointwise a.s., i.e.

$$
\log \frac{d P_{\tilde{Y}_{0}^{T,(\text { snr) }} \mid \tilde{X}_{0}^{T}}}{d P_{\tilde{Y}_{0}^{T,(\text { snr })}}}=\log \frac{d P_{\mathbf{Y}_{\text {snr }} \mid \mathbf{X}}}{d P_{\mathbf{Y}_{\mathrm{snr}}}} \quad \text { a.s. }
$$

We now apply the identity in 160 directly to the log Radon-Nikodym derivative (quantity inside brackets in r.h.s. of (174) to get

$$
\begin{aligned}
\log \frac{d P_{\mathbf{Y}_{\text {snr }} \mid \mathbf{X}}}{d P_{\mathbf{Y}_{\mathrm{snr}}}} & =\frac{1}{2} \int_{0}^{\mathrm{snr}}\|\mathbf{X}-\mathrm{E}[\mathbf{X} \mid \mathbf{Y} ; \gamma]\|^{2} d \gamma+\int_{0}^{\mathrm{snr}}\left(\mathbf{X}-\mathrm{E}\left[\mathbf{X} \mid \mathbf{Y}_{\gamma}\right]\right) \cdot d \mathbf{W}_{\gamma} \\
& =\frac{1}{2} \int_{0}^{\mathrm{snr}} \int_{0}^{T}\left(\tilde{X}_{t}-\mathrm{E}\left[\tilde{X}_{t} \mid \tilde{Y}_{0}^{T} ; \gamma\right]\right)^{2} d t d \gamma+\int_{0}^{\mathrm{snr}}\left(\mathbf{X}-\mathrm{E}\left[\mathbf{X} \mid \mathbf{Y}_{\gamma}\right]\right) \cdot d \mathbf{W}_{\gamma} \\
& =\frac{1}{2} \int_{0}^{\mathrm{snr}} \operatorname{sse}(\mathrm{T}, \gamma) d \gamma+\int_{0}^{\mathrm{snr}} \sum_{i=1}^{M}\left(X_{i}-\mathrm{E}\left[X_{i} \mid \mathbf{Y} ; \gamma\right]\right) d W_{\gamma}^{[i]}
\end{aligned}
$$

where sse $(\mathrm{T}, \gamma)$ represents the squared smoothing error in an observation window of duration $\mathrm{T}$ at signal to noise ratio $\gamma$. Note that $\mathrm{E}[\operatorname{sse}(\mathrm{T}, \gamma)]=\operatorname{mmse}(\gamma)$. 
We can write the difference as,

$$
\begin{aligned}
N(T) \triangleq \log \frac{d P_{\tilde{Y}_{0}^{T,(\mathrm{snr})} \mid \tilde{X}_{0}^{T}}}{d P_{\tilde{Y}_{0}^{T,(\mathrm{snr})}}}-\frac{1}{2} \int_{0}^{\mathrm{snr}} \operatorname{sse}(\mathrm{T}, \gamma) d \gamma & =\int_{0}^{\mathrm{snr}} \sum_{i=1}^{M}\left(X_{i}-\mathrm{E}\left[X_{i} \mid \mathbf{Y} ; \gamma\right]\right) d W_{\gamma}^{[i]} \\
& =\sum_{i=1}^{M} \int_{0}^{\mathrm{snr}}\left(X_{i}-\mathrm{E}\left[X_{i} \mid \tilde{Y}_{0}^{T} ; \gamma\right]\right) d W_{\gamma}^{[i]}
\end{aligned}
$$

Note that by Itô's Isometry property,

$$
\begin{aligned}
\operatorname{Var}(N(T)) & =\int_{0}^{\mathrm{snr}} \mathrm{E}\left[\|\mathbf{X}-\mathrm{E}[\mathbf{X} \mid \mathbf{Y} ; \gamma]\|^{2}\right] d \gamma \\
& =\int_{0}^{\mathrm{snr}} \mathrm{E}\left[\int_{0}^{T}\left(\tilde{X}_{t}-\mathrm{E}\left[\tilde{X}_{t} \mid \tilde{Y}_{0}^{T} ; \gamma\right]\right)^{2} d t\right] d \gamma \\
& =\int_{0}^{\mathrm{snr}} \operatorname{mmse}(\gamma) \stackrel{(f)}{=} 2 I(\mathrm{snr}),
\end{aligned}
$$

which is also an interesting result. Note that (f) follows from the continuous-time I-MMSE relationship. We now argue that the result that we just established for piecewise constant processes carries through for general continuous time processes with finite average power. We refer the reader to [4, Section IV.C] for details in making this approximation, and provide the sketch below.

The main idea is to induce a stepwise process $X_{0}^{(n), T}$ defined by

$$
X_{t}^{(n)} \equiv \frac{1}{2^{n} T} \int_{i 2^{-n} T}^{(i+1) 2^{-n} T} X_{t} d t \text { for } t \in\left(i 2^{-n} T,(i+1) 2^{-n} T\right] .
$$

Since processes in $P$ have finite energy, the integral $\int_{i 2^{-n} T}^{(i+1) 2^{-n} T} X_{t} d t$ exists and is finite P-a.s. and is in $L_{2}(P)$. We now note that,

$$
X_{0}^{(n), T} \stackrel{n \rightarrow \infty}{\rightarrow} X_{0}^{T} \text { in } L^{2}(d t d P) .
$$

Further, the Radon-Nikodym derivates of the induced measures in the stepwise process converge to the actual Radon-Nikodym derivates in a $P_{Y_{0}^{T}}$-a.s. sense. Therefore, the approximation allows us to generalize our results to the class of all finite power continuous time processes.

Duncan's theorem proves as a corollary, the equivalence of the causal and anti-causal squared errors. We now present a proof for Proposition 5, where we establish a pointwise version of the result.

Proof of Proposition 5. We first note the following relationship which follows directly from Theorem 3 ,

$$
\log \frac{d P_{Y_{0}^{T} \mid X_{0}^{T}}}{d P_{Y_{0}^{T}}}=\frac{1}{2} \int_{0}^{T}\left(X_{t}-\mathrm{E}\left[X_{t} \mid Y_{0}^{t}\right]\right)^{2} d t+\int_{0}^{T}\left(X_{t}-E\left[X_{t} \mid Y_{0}^{t}\right]\right) \cdot d W_{t} \quad \text { a.s. }
$$

Let us recall the transformations defined in 62 - 64. Note in addition, that $\mathrm{E}\left[\tilde{X}_{t} \mid \tilde{Y}_{0}^{t}\right]$ is adapted to the filtration induced by $\left(\tilde{X}^{T}, B^{T}\right)$, so

$$
\int_{0}^{T}\left(\tilde{X}_{t}-E\left[\tilde{X}_{t} \mid \tilde{Y}_{0}^{t}\right]\right) \cdot d B_{t}
$$

is well defined in the standard sense of an Itô integral. 
Applying the relation in 185, with the associations $X^{T} \rightarrow \tilde{X}^{T}, W^{T} \rightarrow B^{T}$ and $Y^{T} \rightarrow \tilde{Y}^{T}$, gives us

$$
\log \frac{d P_{\tilde{Y}_{0}^{T} \mid \tilde{X}_{0}^{T}}}{d P_{\tilde{Y}_{0}^{T}}}=\frac{1}{2} \int_{0}^{T}\left(\tilde{X}_{t}-\mathrm{E}\left[\tilde{X}_{t} \mid \tilde{Y}_{0}^{t}\right]\right)^{2} d t+\int_{0}^{T}\left(\tilde{X}_{t}-E\left[\tilde{X}_{t} \mid \tilde{Y}_{0}^{t}\right]\right) \cdot d B_{t} \quad \text { a.s. }
$$

On the other hand, since there are one-to-one transformations to get from $\tilde{X}^{T}$ to $X^{T}$ and from $\tilde{Y}^{T}$ to $Y^{T}$, we have

$$
\log \frac{d P_{Y_{0}^{T} \mid X_{0}^{T}}}{d P_{Y_{0}^{T}}}=\log \frac{d P_{\tilde{Y}_{0}^{T} \mid \tilde{X}_{0}^{T}}}{d P_{\tilde{Y}_{0}^{T}}} \text { a.s. }
$$

Combining (188), (187) and (185), we get the following equality (in the almost sure sense):

$\frac{1}{2} \int_{0}^{T}\left(X_{t}-\mathrm{E}\left[X_{t} \mid Y_{0}^{t}\right]\right)^{2} d t-\frac{1}{2} \int_{0}^{T}\left(\tilde{X}_{t}-\mathrm{E}\left[\tilde{X}_{t} \mid \tilde{Y}_{0}^{t}\right]\right)^{2} d t=\int_{0}^{T}\left(\tilde{X}_{t}-E\left[\tilde{X}_{t} \mid \tilde{Y}_{0}^{t}\right]\right) \cdot d B_{t}-\int_{0}^{T}\left(X_{t}-E\left[X_{t} \mid Y_{0}^{t}\right]\right) \cdot d W_{t}$

Simplifying the above expression further, we get

$$
\begin{aligned}
\frac{1}{2} \int_{0}^{T}\left(X_{t}-\mathrm{E}\left[X_{t} \mid Y_{0}^{t}\right]\right)^{2} d t-\frac{1}{2} \int_{0}^{T}\left(\tilde{X}_{t}-\mathrm{E}\left[\tilde{X}_{t} \mid \tilde{Y}_{0}^{t}\right]\right)^{2} d t & =\int_{0}^{T}\left(\tilde{X}_{t}-E\left[\tilde{X}_{t} \mid \tilde{Y}_{0}^{t}\right]\right) \cdot d B_{t}-\int_{0}^{T}\left(X_{t}-E\left[X_{t} \mid Y_{0}^{t}\right]\right) \cdot d W_{t} \\
& \left.=\int_{0}^{T} E\left[X_{t} \mid Y_{0}^{t}\right] d W_{t}-\int_{0}^{T} E\left[\tilde{X}_{t} \mid \tilde{Y}_{0}^{t}\right]\right) d B_{t}
\end{aligned}
$$

where the second equality holds true for all processes that satisfy the following benign condition in 192 :

$$
\int_{0}^{T} \tilde{X}_{t} d B_{t}=\int_{0}^{T} X_{t} d W_{t} \quad \text { a.s. }
$$

Note that 192 is simple to verify for the class of piecewise constant processes on the interval $[0, T]$. By approximation arguments similar to the ones given in the proof of Theorem 10, one can establish the equality (192) for all finite power continuous time processes.

Having established the pointwise I-MMSE relationship for continuous-time processes, we now present a pointwise version of the causal vs. non-causal error relationship discussed in Subsection II-B5 In doing so, for simplicity, we restrict our attention to the class of piecewise-constant processes. Our characterization is valid for all piecewise constant processes, and (appealing to the arguments given in the proof of Theorem 10p therefore holds for all continuous time processes that can be approxmiated as such.

Let $\tilde{X}_{0}^{T}$ denote a piecewise-constant process, such that

$$
\tilde{X}_{t} \equiv X_{i} \quad \text { for all } \frac{(i-1)}{M} T \leq t \leq \frac{i}{M} T,
$$

where $X_{i} \sim P$, and $1 \leq i \leq M$. We fix snr=1, and let the output be denoted by $\left\{\tilde{Y}_{t}^{(\gamma)}\right\}_{0 \leq t \leq T}$ for the channel described in 52. I.e.,

$$
d \tilde{Y}_{t}^{(\gamma)}=\gamma \tilde{X}_{t} d t+d W_{t}^{(\gamma)}
$$

for $\gamma \in[0,1]$ and $t \in[0, T]$. We first note from Proposition 3 that the input-output information density can be written as

$$
\log \frac{d P_{\tilde{Y}_{0}^{T,(1)} \mid \tilde{X}_{0}^{T}}}{d P_{\tilde{Y}_{0}^{T,(1)}}}=\frac{1}{2} \int_{0}^{T}\left(\tilde{X}_{t}-\mathrm{E}\left[\tilde{X}_{t} \mid \tilde{Y}_{0}^{t,(1)}\right]\right)^{2} d t+\int_{0}^{T}\left(\tilde{X}_{t}-E\left[\tilde{X}_{t} \mid \tilde{Y}_{0}^{t,(1)}\right]\right) \cdot d W_{t}^{(1)} \quad \text { a.s. }
$$


Recall the definitions of $\mathbf{X}, \mathbf{Y}_{\gamma}$ and $\mathbf{W}_{\gamma}$ in the discussion following [173] in the proof of Theorem 10 Also, in establishing Theorem 10, we derive a relationship for the input-output information density from (174) and (176), namely:

$$
\log \frac{d P_{\tilde{Y}_{0}^{T,(1)} \mid \tilde{X}_{0}^{T}}}{d P_{\tilde{Y}_{0}^{T,(1)}}}=\frac{1}{2} \int_{0}^{1} \int_{0}^{T}\left(\tilde{X}_{t}-\mathrm{E}\left[\tilde{X}_{t} \mid \tilde{Y}_{0}^{T,(1)}\right]\right)^{2} d t d \gamma+\int_{0}^{1}\left(\mathbf{X}-\mathrm{E}\left[\mathbf{X} \mid \mathbf{Y}_{\gamma}\right]\right) \cdot d \mathbf{W}_{\gamma} \quad \text { a.s. }
$$

We now combine (195) and 196 to write down the pointwise characterization of the filtering and smoothing errors,

$$
\begin{gathered}
\int_{0}^{T}\left(\tilde{X}_{t}-\mathrm{E}\left[\tilde{X}_{t} \mid \tilde{Y}_{0}^{t,(1)}\right]\right)^{2} d t-\int_{0}^{1} \int_{0}^{T}\left(\tilde{X}_{t}-\mathrm{E}\left[\tilde{X}_{t} \mid \tilde{Y}_{0}^{T,(\gamma)}\right]\right)^{2} d t d \gamma= \\
\int_{0}^{1} 2\left(\mathbf{X}-\mathrm{E}\left[\mathbf{X} \mid \mathbf{Y}_{\gamma}\right]\right) \cdot d \mathbf{W}_{\gamma}-\int_{0}^{T} 2\left(\tilde{X}_{t}-E\left[\tilde{X}_{t} \mid \tilde{Y}_{0}^{t,(1)}\right]\right) \cdot d W_{t}^{(1)} \quad \text { a.s. }
\end{gathered}
$$

Thus, the difference between the filtering and smoothing errors has an explicit characterization in terms of stochastic integrals. It is instructive to note that taking expectation on both sides of (197) establishes the identity in 60, for snr=1, by using the fact that the Itô integrals in the r.h.s. of (197) have zero mean.

We now present proofs of results stated in Section $\mathrm{III}$ In Proposition 6 , we established that $\mathrm{E}[Z \mid X]=0$, for all underlying distributions of the signal $X$ that have finite variance. The proof invokes the setting in [3] and is presented below.

Proof of Proposition 6. We denote the mean squared error due to mismatch at signal to noise ratio $\gamma>0$, by $\operatorname{mse}_{P, Q}(\gamma)$ defined in [14. Note from [3, Section V.] that we have,

$$
\begin{aligned}
I(X ; \sqrt{\operatorname{snr} X}+N) & =\int D(N+\sqrt{\operatorname{snr}} x \| N+\sqrt{\operatorname{snr}} X) d P_{X}(x) \\
& =\int D\left(\delta_{x} * \mathcal{N}\left(0, \operatorname{snr}^{-1}\right) \| P_{X} * \mathcal{N}\left(0, \operatorname{snr}^{-1}\right)\right) d P_{X}(x) \\
& =\frac{1}{2} \iint_{0}^{\mathrm{snr}} \operatorname{mse}_{P_{x}, \delta_{x}}(\gamma)-\operatorname{mmse}_{\delta_{x}}(\gamma) d \gamma d P_{X} \\
& =\frac{1}{2} \iint_{0}^{\mathrm{snr}} \operatorname{mse}_{P_{x}, \delta_{x}}(\gamma) d \gamma d P_{X} \\
& =\frac{1}{2} \int_{0}^{\mathrm{snr}} \operatorname{mmse}(\gamma) d \gamma
\end{aligned}
$$

From (199) and 201] it is clear that

$$
\begin{gathered}
\mathrm{E}\left[\log \frac{\left.d P_{Y_{\mathrm{snr}} \mid X} \mid X\right]}{d P_{Y_{\mathrm{snr}}}} \mid X\right]\left[\frac{1}{2} \int_{0}^{\mathrm{snr}}\left(X-\mathrm{E}\left[X \mid Y_{\gamma}\right]\right)^{2} d \gamma \mid X\right] \\
\Longrightarrow E[Z \mid X]=0 \text { a.s. } \\
I_{1}=\frac{1}{2} \log (1+\mathrm{snr})+\frac{1}{2}\left(\frac{Y^{2}}{1+\mathrm{snr}}-\left(Y-\sqrt{\mathrm{snr} X)^{2}}\right)\right. \\
=\frac{1}{2} \log (1+\mathrm{snr})+\frac{1}{2}\left(\frac{\mathrm{snr}}{1+\mathrm{snr}}\left(X^{2}-N^{2}\right)+2 X N \frac{\sqrt{\mathrm{snr}}}{1+\mathrm{snr}}\right)
\end{gathered}
$$


Also,

$$
\mathrm{E}\left[I_{1} \mid X\right]=\frac{1}{2} \log (1+\mathrm{snr})+\frac{1}{2}\left(\frac{\mathrm{snr}}{1+\mathrm{snr}}\left(X^{2}-1\right)\right)
$$

for all underlying processes $P_{X}$. Thus, not only are the random quantities above equal in expectation, but they are also equal in conditional expectation on $X$.

We now turn our attention to the alternative coupling discussed in Section III-B. In Lemma 11, we derived an expression for the pointwise tracking error for the Additive Standard Gaussian coupling 78 for a general input signal $X$. In the following, we present a proof of Lemma 11 .

\section{Proof of Lemma 11 .}

Let us assume $X$ is distributed according to $P_{X}$. Let $f_{Z}(z)$ denote the probability density function of a standard Gaussian random variable. Note for the setting in 78 that,

$$
P_{Y_{\gamma} \mid X}(y \mid x)=\frac{1}{\sqrt{2 \pi}} e^{-\frac{1}{2}(y-\sqrt{\gamma} x)^{2}}=f_{Z}(y-\sqrt{\gamma} x),
$$

and

$$
P_{Y_{\gamma}}(y)=\int P_{Y_{\gamma} \mid X}(y \mid \tilde{x}) d P_{\tilde{x}}
$$

Then,

$$
I_{1}\left(X, Y_{\gamma}\right)=\log \frac{P_{Y_{\gamma} \mid X}\left(Y_{\gamma} \mid X\right)}{P_{Y_{\gamma}}\left(Y_{\gamma}\right)}
$$

We now look at the differential form of the I-MMSE relationship. Namely,

$$
\frac{\partial I\left(X ; Y_{\gamma}\right)}{\partial \gamma}=\frac{1}{2} \operatorname{mmse}(\gamma)
$$

or its pointwise equivalent

$$
\mathrm{E}\left[\frac{\partial}{\partial \gamma} \log \frac{P_{Y_{\gamma} \mid X}\left(Y_{\gamma} \mid X\right)}{P_{Y_{\gamma}}\left(Y_{\gamma}\right)}-\frac{1}{2}\left(X-\mathrm{E}\left[X \mid Y_{\gamma}\right]\right)^{2}\right]=0 .
$$

Define

$$
\tilde{Z}_{\gamma}=\frac{\partial}{\partial \gamma} \log \frac{P_{Y_{\gamma} \mid X}\left(Y_{\gamma} \mid X\right)}{P_{Y_{\gamma}}\left(Y_{\gamma}\right)}-\frac{1}{2}\left(X-\mathrm{E}\left[X \mid Y_{\gamma}\right]\right)^{2} .
$$

Differentiating 210 w.r.t. snr, we have

$$
\begin{aligned}
\frac{\partial}{\partial \gamma} \log \frac{P_{Y_{\gamma} \mid X}\left(Y_{\gamma} \mid X\right)}{P_{Y_{\gamma}}\left(Y_{\gamma}\right)} & =\frac{\partial}{\partial \gamma} \log P_{Y_{\gamma} \mid X}\left(Y_{\gamma} \mid X\right)-\frac{\partial}{\partial \gamma} \log P_{Y_{\gamma}}\left(Y_{\gamma}\right) \\
& =0-\frac{\partial}{\partial \gamma} \log P_{Y_{\gamma}}\left(Y_{\gamma}\right) \\
& =\frac{1}{P_{Y_{\gamma}}\left(Y_{\gamma}\right)} \int \frac{1}{\sqrt{2 \pi}} e^{-\frac{1}{2}\left(Y_{\gamma}-\sqrt{\gamma} \tilde{x}\right)^{2}}\left(Y_{\gamma}-\sqrt{\gamma} \tilde{x}\right)((X-\tilde{x}) / 2 \sqrt{\gamma}) d P_{\tilde{x}} \\
& =\frac{1}{2 \sqrt{\gamma}} \int \frac{P_{Y_{\gamma} \mid X}\left(Y_{\gamma} \mid \tilde{x}\right)}{P_{Y_{\gamma}}\left(Y_{\gamma}\right)}\left(X Y_{\gamma}-Y_{\gamma} \tilde{x}-\sqrt{\gamma} X \tilde{x}+\sqrt{\gamma} \tilde{x}^{2}\right) d P_{\tilde{x}} \\
& =\frac{1}{2 \sqrt{\gamma}}\left[X Y_{\gamma}-\left(\sqrt{\gamma} X+Y_{\gamma}\right) \mathrm{E}\left[X \mid Y_{\gamma}\right]+\sqrt{\gamma} \mathrm{E}\left[X^{2} \mid Y_{\gamma}\right]\right],
\end{aligned}
$$


where:

- 215$) \Leftarrow P_{Y_{\gamma} \mid X}\left(Y_{\gamma} \mid X\right)=f_{Z}\left(Y_{\gamma}-\sqrt{\gamma} X\right)=f_{Z}(N)$ (and hence does not depend on $\gamma$ )

- 216 $\Leftarrow Y_{\gamma}=\sqrt{\gamma} X+N$ is the explicit dependence of $Y_{\gamma}$ on $\gamma$. Using this, and differentiating with respect to $\gamma$, we obtain the required expression

- $218 \Leftarrow$ the integral is with respect to the conditional law $X \mid Y_{\gamma}$, with $\tilde{x}$ as the variable of integration, while keeping $X$ and $Y_{\gamma}$ constant.

Combining (212), 218) and (213), we get

$$
\begin{aligned}
\tilde{Z}_{\gamma} & =\frac{\partial I_{1}}{\partial \gamma}-\frac{1}{2}\left(X-\mathrm{E}\left[X \mid Y_{\gamma}\right]\right)^{2} \\
& =\frac{1}{2 \sqrt{\gamma}}\left[X Y_{\gamma}-\left(\sqrt{\gamma} X+Y_{\gamma}\right) \mathrm{E}\left[X \mid Y_{\gamma}\right]+\sqrt{\gamma} \mathrm{E}\left[X^{2} \mid Y_{\gamma}\right]\right]-\frac{1}{2}\left(X-\mathrm{E}\left[X \mid Y_{\gamma}\right]\right)^{2} \\
& =\frac{1}{2}\left\{\mathrm{E}\left[X^{2} \mid Y_{\gamma}\right]-X \mathrm{E}\left[X \mid Y_{\gamma}\right]-\left(X-\mathrm{E}\left[X \mid Y_{\gamma}\right]\right)^{2}+\frac{Y_{\gamma}}{\sqrt{\gamma}}\left(X-\mathrm{E}\left[X \mid Y_{\gamma}\right]\right)\right\}
\end{aligned}
$$

Note now that $Z$ according to Definition 1 is

$$
\begin{aligned}
Z & =\log \frac{P_{Y_{\mathrm{snr}} \mid X}\left(Y_{\mathrm{snr}} \mid X\right)}{P_{Y_{\mathrm{snr}}}\left(Y_{\mathrm{snr}}\right)}-\frac{1}{2} \int_{0}^{\mathrm{snr}}\left(X-\mathrm{E}\left[X \mid Y_{\gamma}\right)^{2} d \gamma\right. \\
& =\int_{0}^{\mathrm{snr}} \tilde{Z}_{\gamma} d \gamma .
\end{aligned}
$$

\section{CONCLUSION}

We consider the scenario of mean square estimation of a signal observed through additive white Gaussian noise. We formulate classical information and estimation relationships in these contexts as expectation identities. We explicitly characterize the input-output information density for both scalar and continuous time Gaussian channels. Using this characterization, which relies on Girsanov theory, we obtain pointwise representations of these identities with the expectations removed and discover that these random quantities also have classical information-estimation links. In particular, canonical measures of information and estimation appear to be bridged by the second moment of the pointwise tracking error between the information density and the scaled filtering error. In this manner we present pointwise relations for Duncan's theorem, mismatched estimation, channels with feedback, the I-MMSE relationship as well as the causal vs. non-causal and causal vs. anticausal errors. A special treatment for scalar estimation is also provided where we present and discuss alternative couplings to the Brownian motion corrupted channel. We also provide applications of these results to obtain new and interesting relations in the information-estimation arena.

The first and second moments of the tracking error in the Gaussian setting have direct implications on information and estimation relations. In future work,we would like to see whether similar implications emerge for higher order moments as well. In addition it would be interesting to investigate whether pointwise relationships similar to the Gaussian case, hold also for the Poissonian channel, where links between estimation and information have been recently uncovered in [15] for a natural loss function. 


\section{ACKNOWLEDGEMENT}

The authors thank Rami Atar for valuable discussions. This work has been supported under a Stanford Graduate Fellowship, NSF grant CCF-0729195, and the Center for Science of Information (CSoI), an NSF Science and Technology Center, under grant agreement CCF-0939370.

\section{REFERENCES}

[1] T. E. Duncan, "On the calculation of Mutual Information," SIAM J. Appl. Math., vol. 19, pp. 215-220, Jul. 1970.

[2] D. Guo, S. Shamai and S. Verdú, "Mutual Information and minimum mean-square error in Gaussian channels", IEEE Trans. Information theory, vol. IT-51, no. 4, pp.1261-1283, Apr. 2005.

[3] S. Verdú, "Mismatched Estimation and relative Entropy", IEEE Trans. Information theory, vol 56., no. 8, pp. 3712-3720, Aug. 2010.

[4] T. Weissman, "The Relationship Between Causal and Noncausal Mismatched Estimation in Continuous-Time AWGN Channels", IEEE Trans. Information theory, vol. 56, no. 9, pp. 4256 - 4273, September 2010.

[5] T.T. Kadota, M. Zakai, J. Ziv, "Mutual Information of the White. Gaussian Channel With and Without Feedback", IEEE Transactions on Information theory, vol. IT-17, no. 4, July 1971.

[6] Y. Polyanskiy, H. V. Poor, S. Verdú, "New Channel Coding Achievability Bounds", IEEE Int. Symposium on Information Theory 2008, Toronto, Ontario, Canada, July 6-11, 2008

[7] I. Karatzas and A. E. Shreve, Brownian Motion and Stochastic Calculus, 2nd ed. Springer-Verlag, New York, 1988.

[8] T. Weissman, Y.-H. Kim, H. H. Permuter, "Directed Information, Causal Estimation, and Communication in Continuous Time", submitted to IEEE Transactions on Information theory, Sep. 2011.

[9] M. Zakai, "On Mutual Information, Likelihood Ratios, and Estimation Error for the Additive Gaussian Channel", IEEE Transactions on Information theory, vol. 51, No. 9, September 2005.

[10] D. Guo, "Gaussian Channels: Information, Estimation and Multiuser Detection," Ph.D. Thesis, Princeton University, 2004

[11] T. Kailath,"The structure of Radon-Nykodim derivatives with respect to Wiener and related measures," Ann. Math. Statist., vol. 42, no. 3, pp. 1054-1067, 1971.

[12] I. V. Girsanov, "On transforming a certain class of stochastic processes by absolutely continuous substitution of measures," Theory Probab. Appl., vol. 5, pp. 285 301, 1960.

[13] J. M. Steele, Stochastic Calculus and Financial Applications, Springer, 2010.

[14] T. Kailath, "A General Likelihood-Ratio Formula for Random Signals in Gaussian Noise", IEEE Transactions on Information theory, vol. IT-15, No. 3, May 1969.

[15] R. Atar, T. Weissman, "Mutual Information, Relative Entropy, and Estimation in the Poisson Channel", IEEE Transactions on Information theory, vol. 58, no. 3, March 2012. 